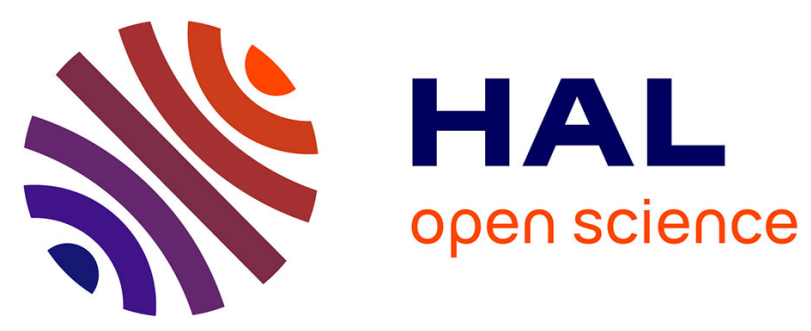

\title{
Three-dimensional finite element thermomechanical modeling of additive manufacturing by selective laser melting for ceramic materials
}

Qiang Chen, Gildas Guillemot, Charles-André Gandin, Michel Bellet

\section{- To cite this version:}

Qiang Chen, Gildas Guillemot, Charles-André Gandin, Michel Bellet. Three-dimensional finite element thermomechanical modeling of additive manufacturing by selective laser melting for ceramic materials. Additive Manufacturing, 2017, 16, pp.124 - 137. 10.1016/j.addma.2017.02.005 . hal01552410

HAL Id: hal-01552410

https://hal-mines-paristech.archives-ouvertes.fr/hal-01552410

Submitted on 2 Jul 2017

HAL is a multi-disciplinary open access archive for the deposit and dissemination of scientific research documents, whether they are published or not. The documents may come from teaching and research institutions in France or abroad, or from public or private research centers.
L'archive ouverte pluridisciplinaire HAL, est destinée au dépôt et à la diffusion de documents scientifiques de niveau recherche, publiés ou non, émanant des établissements d'enseignement et de recherche français ou étrangers, des laboratoires publics ou privés. 


\title{
Three-dimensional finite element thermomechanical modeling of additive manufacturing by selective laser melting for ceramic materials
}

\author{
Qiang Chen, Gildas Guillemot, Charles-André Gandin and Michel Bellet ${ }^{*}$ \\ MINES ParisTech, PSL Research University, CEMEF - Centre de mise en forme des \\ matériaux, CNRS UMR 7635, CS 10207 rue Claude Daunesse, 06904 Sophia Antipolis
}

Cedex, France

\begin{abstract}
A model for additive manufacturing by selective laser melting of a powder bed with application to alumina ceramic is presented. Based on Beer-Lambert law, a volume heat source model taking into account the material absorption is derived. The level set method is used to track the shape of deposed bead. An energy solver is coupled with thermodynamic database to calculate the melting-solidification path. Shrinkage during consolidation from powder to liquid and compact medium is modeled by a compressible Newtonian constitutive law. A semi-implicit formulation of surface tension is used, which permits a stable resolution to capture the liquid/gas interface. The influence of different process parameters on temperature distribution, melt pool profiles and bead shapes is discussed. The effects of liquid viscosity and surface tension on melt pool dynamics are investigated. Three dimensional simulations of several passes are also presented to study the influence of the scanning strategy.
\end{abstract}

Keywords: additive manufacturing, selective laser melting, level set, compressible, surface tension, ceramic

\section{Introduction}

In recent years, additive manufacturing (AM) has met a growing interest from various industrial sectors because of its advantages compared with conventional forming techniques. $\mathrm{AM}$ is often referred to as " $3 \mathrm{D}$ printing" and gives the possibility to design parts with complex geometry. AM offers the opportunity to manufacture a large variety of objects for a wide range of practical applications. Prototypes for testing can be printed right after the design rather than waiting for a long time, thus providing a more efficient strategy than usual industrial practice based on casting, machining or other forming processes. Among other

\footnotetext{
* Corresponding author

Tel.: +33 (0) 493957461

Email address: michel.bellet@mines-paristech.fr
} 
advantages, assemblies can be directly printed into a single final product. Moreover, redesign of part shape with equivalent mechanical performance can be also proposed. However, most of AM processes are much slower than conventional technologies, specific defects such as cracks often exist and poor surface quality is usually encountered, all resulting in poor control of the mechanical properties [1]. As a consequence, considerable efforts must be made on the setting of AM parameters in order to manage the quality and in-service properties of AM parts.

Different AM processes have been exploited with application to various materials. Among them, Selective Laser Melting (SLM) has drawn peoples' attention, especially in the fields of aerospace and medical orthopedic for metallic alloys hard to shape with conventional technologies. Applications in aluminum [1], stainless steel [2], titanium [3],[4], cobalt chrome [5] and nickel [6] are of particular interest. A typical working cycle for SLM is shown in Figure 1 [7]. At first, a vertical build piston moves downward an upper shelf by a layer thickness. The layer thickness varies from $20 \mu \mathrm{m}$ to $100 \mu \mathrm{m}$, considering a balance between fine resolution and good powder flowability [8]. The feed piston alternatively moves powder upward more than a layer thickness. A roller then shoves the powder horizontally into the build chamber with downward vertical press. A laser beam with focused spot diameter is employed to selectively melt powder, according to predefined trajectories generated from a three-dimensional CAD model. The molten layer is solidified and bonded to the previous one in order to progressively build a compact part. The whole process is usually conducted under a protective atmosphere in order to avoid oxidation. Full melting of the powder is targeted for SLM, making a difference with selective laser sintering (SLS) [9] which binds powder without totally melting the material. As a consequence, fully dense and near net shape components can be obtained by SLM, resulting into better mechanical properties [10].

Alumina $\left(\mathrm{Al}_{2} \mathrm{O}_{3}\right)$ and zirconia $\left(\mathrm{ZrO}_{2}\right)$ ceramics are widely used due to their outstanding mechanical strength and excellent thermal and wear resistances [10], which is especially attractive to aeronautic industry. Traditional manufacturing processes often suffer from high cost, high tool wear (machining), shrinkage (sintering) and constraints of part geometry. SLM could open a door to a larger application of ceramics. Compared with metals, some special attention should be paid to ceramics in SLM. Ceramics have an absorption coefficient for long infrared laser wavelength which is several orders of magnitude lower than that of metals [11]. This leads to totally different temperature distribution and shape of melt pool as laser radiation can penetrate deeper into the material. Another important aspect is cracking due to thermal stresses. However, Hagedorn et al. [10] could totally eliminate cracks by hightemperature preheating just below the melting point of material and thus, in principle, offer a practical solution to this issue.

The mechanical properties of final parts are tightly related to thermal evolution during manufacturing. Large heating and cooling rates happen locally around the laser spot in a small 
region, leading to phase transformation in a high thermal gradient difficult to tailor by experimental studies. In order to optimize process parameters, numerical models are currently proposed for AM processes. Considering the generality of the modeling approaches, the literature study has not to be limited to SLM. Two categories can be distinguished, corresponding to the macro-scale of the parts and to the meso-scale of the bead. For large scale modeling, Hodge et al. [12] chose a complex heat source model described by Gusarov et al. [13]. The temperature field was predicted, as well as the shape of the melt pool, for $316 \mathrm{~L}$ stainless steel. Configuration with a melt pool propagated above unconsolidated powder was predicted, caused by the insulating behavior of the powder bed. King et al. [14] pursued the work of Hodge et al. [12] to predict the temperature and stress in a $6 \mathrm{~cm}$ tall prism part of 316L stainless steel. Residual stresses were found to compare favorably with experiments. Li and $\mathrm{Gu}$ [15] predicted the temperature field in a titanium powder bed, with a Gaussian heat source model and temperature dependent thermal properties. They found that most heat was eliminated through conduction in the cold substrate and that the material experienced a rapid quenching process. Roberts et al. [16] used a heat source model considering the absorption of laser energy described in [17] and employed the method of element birth and death to simulate the addition of multiple layers, for Ti-6Al-4V material. The same method was used by Marion et al.[18] for Direct Metal Deposition.

Modeling at scale of bead helps to understand the complex temperature distribution and the shape of the melt pool. By varying process parameters, one can predict a working region in which stable bead shape can be obtained. King et al. [14] simulated the bead formation with and without surface tension, by using a powder model. They found that surface tension has a tendency to smooth melt pool and improve heat transfer to substrate. Körner et al. [19] provided a two-dimensional numerical approach based on the lattice Boltzmann model to predict melting and solidification of the powder. Stochastic spatial distribution of the powder particles and capillarity force were investigated. Results showed that the packing density of the powder bed has the most significant effect on the melt pool characteristics. Zhou et al. [20] firstly generated a 3D random packing of spherical particles with same or different size. A ray tracing algorithm based on a Monte Carlo method was then used to simulate radiation heat transfer in bimodal structures.

A numerical Finite Element (FE) model for AM by SLM is presented hereafter. It aims at studying thermomechanical phenomena at the scale of the bead, during unitary and successive multiple passes deposition of ceramic material. The model focuses on heat transfer and fluid flow during melting of the powder bed, resulting in the prediction of the shape of the bead. A 3D application for material deposition in SLM with alumina ceramics is proposed. Considering the relative transparency of this material with respect to laser radiation, a volume heat source model based on the Beer-Lambert law is derived, taking into account the local absorption coefficient. The Level Set (LS) method is used to track the material/gas interface 
and hence the bead shape. Shrinkage due to transformation of powder particles into a compact medium is taken into account by a compressible Newtonian constitutive law. A stable semiimplicit formulation is employed for the surface tension term in the momentum conservation. The solidification stage is modeled considering a prescribed evolution of the phase fraction with temperature. The influence of different process parameters on melt pool profiles and bead shapes is discussed, such as laser power and scanning velocity, or layer thickness of the powder bed. Sensitivity tests are also done regarding material properties, such as absorption, surface tension and viscosity.

\section{Modeling}

The system is made of a material domain and a gas domain, as shown in Figure 2a. The material domain hereafter considered is pure alumina. In its initial state, it consists of a powder bed with a certain porosity, located on top of consolidated layers acting as a substrate. This powder material is melted during laser heating and cooled down to form a compact deposition, or consolidated region. During this one-way transformation, densification takes place and is modeled through the variation of the apparent density of the material from powder state to liquid and solid states. Possible states for the compact medium are liquid and solid, only depending on temperature. The surface shape of the melt pool, i.e. the interface between the two domains, material and gas, results from combined effects of forces acting on the liquid. They depend on properties such as liquid viscosity and surface tension at the liquid/gas interface. The evolution of the gas/material interface is tracked by a LS method and mesh adaptation. All conservation equations are established in this two-domain system considering a continuous evolution of the materials properties. The main assumptions of the proposed approach are summarized as follows (additional hypotheses will be introduced and discussed directly in the text):

- The powder is assimilated to a continuum;

- No powder projection (i.e. no powder loss);

- No residual porosity once the powder is fully melted (i.e. no release of dissolved gas to form pores; no gas entrapment).

\subsection{Level Set method}

The system is made of two domains, namely the material domain $\left(D_{1}\right)$ and the gas domain $\left(D_{2}\right)$ as illustrated in Figure 2a. The Level Set (LS) method [21] is used to track the evolution of the material/gas interface. The interface is defined by the isovalue $\psi=0$, where $\psi=\psi(\boldsymbol{X}, t)$ is the signed distance function to the interface of any position $\boldsymbol{X}$ at time $t$ located in the two-domain system. Negative values are arbitrarily chosen in the material $(\psi<0 \forall \boldsymbol{X} \in$ $\left.D_{1}\right)$, thus leading to positive values in the gas $\left(\psi>0 \forall \boldsymbol{X} \in D_{2}\right)$. This interface is shown by 
the black bold line for the interface with the consolidated solid and powder material subdomains and the blue bold line for the interface with the liquid material subdomain. Centered at this interface, we define a transition zone with half thickness $\epsilon$ being a small positive value. A Heaviside function, $\mathcal{H}$, is then defined, which continuously evolves within the transition zone from 0 in the material to 1 in the gas:

$$
\mathcal{H}(\psi)=\left\{\begin{array}{lr}
0 & \begin{array}{l}
\psi<-\epsilon \\
\frac{1}{2}\left[1+\frac{\psi}{\epsilon}+\frac{1}{\pi} \sin \left(\frac{\pi \psi}{\epsilon}\right)\right] \\
\psi \\
\psi
\end{array} \quad|\psi| \leq \epsilon
\end{array}\right.
$$

Averaged properties in each domain, $\langle\chi\rangle^{D_{i}}(i=1,2)$, are computed over the two-domain system by using the Heaviside function, thus defining global averaged properties $\{\chi\}$ [22],[23]:

$$
\{\chi\}=\mathcal{H}\langle\chi\rangle^{D_{2}}+(1-\mathcal{H})\langle\chi\rangle^{D_{1}}
$$

This is used for density, enthalpy, thermal conductivity, etc., and aims at defining a set of equations averaged over the whole system.

The distance function $\psi$ should be recomputed at each time step as the interface position does continuously change. It essentially consists of solving the following transport equation:

$$
\frac{d \psi}{d t}=\frac{\partial \psi}{\partial t}+\boldsymbol{u}^{L S} \cdot \nabla \psi=0
$$

where $\boldsymbol{u}^{L S}$ denotes the velocity of the interface at the boundary between the two domains tracked by the LS method. This is carried out just after the solution of the momentum equation that provides with the velocity field in the two-domain system. However, the transportation of the distance function does not guarantee equality between the geometrical distance $d$ from a point to the interface (except points located at the interface) and the value of $|\psi|$ in the whole simulation domain. Therefore, $\psi$ is recomputed by a geometrical method [24] with respect to the new position of the interface resulting from Eq.(3), considering that the resolution is only valid for the specific transportation of the interface $(\psi=0)$.

\subsection{Governing equations}

\subsubsection{Energy conservation and heat source model}

The temperature distribution during AM by SLM is computed by the resolution of the non-steady energy conservation equation:

$$
\frac{\partial\{\rho h\}}{\partial t}+\nabla \cdot\{\rho h \boldsymbol{u}\}-\nabla \cdot\{\lambda \nabla T\}=\left\{\dot{q}_{L}\right\}-\left\{\dot{q}_{r}\right\}
$$

where $T$ is the temperature, $\rho$ is the density, $h$ is the specific enthalpy and $\lambda$ is the thermal conductivity. The input laser source $\left\{\dot{q}_{L}\right\}$, and radiation loss $\left\{\dot{q}_{r}\right\}$, at the material/gas interface will be detailed in the sequel. The enthalpy of each phase varies with temperature as described 
in [25]. The thermal conductivity in the material is computed based on the properties of the dense matter and the powder as it will be detailed later. The temperature dependency of these parameters is taken into account in the nonlinear energy solver [26].

A Nd:YAG laser beam with wavelength $1064 \mathrm{~nm}$ is currently used in AM by SLM. This equipment provides low interaction with ceramics, meaning that low heat efficiency is taking place. Pure alumina is then said to be "almost transparent" with a characteristic absorption length of $106 \pm 7 \mu \mathrm{m}$ [27]. This means that radiation can propagate deeply in the material, interaction not being restricted to the vicinity of the surface as it is the case for metallic materials where this characteristic length is lower than $0.1 \mu \mathrm{m}$ [11]. Below the heat source, the entire thickness of the ceramic powder bed and several of the previously deposited layers are thus heated by the laser beam. This effect should be avoided or moderated as it causes additional melting-solidification history and more complicated phase transitions, leading to quality problems [28]. In order to increase the absorption of ceramics, dopants such as carbon particles have to be added into the powder bed. Consequently, the involvement of gas, powder particles and dopant particles makes the interaction between the laser and the powder bed very complicated. Although modeling methods at the particle scale exist [20], we propose a continuous approach developed at a larger scale. Consequently interaction between the laser beam and the powder bed at the scale of the powder particles will not be considered and an effective absorption coefficient of the laser energy will be used. Considering the significant effect of the local absorption coefficient, we propose a volume heat source model based on the Beer-Lambert law [11],[29], which describes the attenuation of heat flux in a logarithmic way (here local coordinate system is taken, with $z$ indicating the laser propagation direction as defined in Figure 2a):

$$
\frac{d(\ln (\Phi))}{d z}=-\alpha
$$

where $\Phi$ is the local heat flux which penetrates the material at the depth $z$ computed in the laser beam direction and $\alpha$ is the local absorption coefficient ( $\alpha$ is the inverse of the characteristic absorption length mentioned above). This coefficient can vary depending on the material state and the local phase fractions (liquid phase and/or different solid phases). Assuming that the variation of the heat flux in thickness $d z$ is only due to absorption by the ceramic material, we can get the expression of a corresponding volume heat source by:

$$
\dot{q}_{L}=-\frac{d \Phi}{d z}=\alpha \Phi=\Phi_{z=0} \alpha \exp \left(-\int_{0}^{z} \alpha d l\right)
$$

where $\Phi_{z=0}$ is the initial heat flux at $z=0$ located in the gas domain (Figure 2a). The heat flux of the laser beam is assumed to follow a radial Gaussian distribution with a standard deviation equal to half of the laser radius $R_{L}$ :

$$
\Phi_{r, Z=0}=(1-R) \frac{2 P_{L}}{\pi R_{L}^{2}} \exp \left(-\frac{2 r^{2}}{R_{L}^{2}}\right)
$$


where $P_{L}$ is the nominal laser power and $R$ is the reflection coefficient, usually much lower than the ones encountered in metallic materials [8]. Note that the integration of $\Phi_{r, z=0}$ from 0 to $R_{L}$ is $86 \%$ of the total effective laser power $(1-R) P_{L}$. The volume heat source model can be finally expressed by:

$$
\dot{q}_{L}(r, z)=(1-R) \frac{2 P_{L}}{\pi R_{L}^{2}} \exp \left(-\frac{2 r^{2}}{R_{L}^{2}}\right) \alpha \exp \left(-\int_{0}^{z} \alpha d l\right)
$$

The integration of Eq.(8) along the z-direction should take into account the absorption variation depending on material properties and temperature. As laser energy can never be totally absorbed within a finite depth, numerical implementation requires truncation of the penetration depth. This is achieved by defining a depth where $90 \%$ of the energy is absorbed. A regular grid covering the region impacted by the laser is introduced to compute the integral of Eq.(8) as schematized in Figure 2b. The absorption value of each mesh node is firstly interpolated on the grid points. Then we can approximate easily the integral by trapezoidal rule. Finally, the integral term at the grid points is interpolated onto the mesh nodes.

The radiation loss $\dot{q}_{r}$ must be imposed as a boundary condition at the material/gas interface. However, with the LS method, this interface is immersed in the system. As this interface is not explicitly described and evolves with time, it is not possible to impose such a boundary condition directly. Here, the Continuum Surface Force (CSF) method developed by Brackbill et al. [30] to transform a surface heat flux into a volume source term is applied. The surface condition is multiplied by the discrete Dirac function, $\delta$. Assuming a pure radiative heat exchange, the heat loss from the surface at temperature $T$ is expressed by:

$$
\dot{q}_{r}=\delta \varepsilon_{r} \sigma\left(T^{4}-T_{0}^{4}\right)
$$

where $\delta=\partial \mathcal{H} / \partial \psi, \sigma$ is the Stefan-Boltzmann constant and $T_{0}$ is the ambient temperature. Here $\varepsilon_{r}$ is the emissivity of the powder bed surface [31].

\subsubsection{Momentum conservation}

In the initial state of AM by SLM, gas occupies the interstices between powder particles (it typically represents $50 \%$ of the powder bed volume). When powder particles are melted, liquid fulfills the interstices alongside with gas evacuation. However, as the interaction between laser and powder is fast and the solidification velocity is very high, gas may be blocked in the consolidated part, resulting into residual porosity in the part [32]. As the powder bed is hereafter considered as a continuum, the inter-particle gas flow and its influence on the liquid movement due to the shear stress (hydrodynamic drag) are not taken into account, together with possible material vaporization and projection. Shrinkage due to the evacuation of the gas from the powder bed is modeled by increasing the apparent density from powder to consolidated part. When liquid forms, surface tension at the liquid/gas interface begins to change the melt pool geometry, which has a significant effect on the final shape of the bead. Combined effects of viscosity, surface tension, gravity, and inertia lead to the 
movement of the material/gas interface. The velocity of the material and gas flows can be obtained by the resolution of the momentum conservation equation:

$$
\{\rho\}\left(\frac{\partial\{\boldsymbol{u}\}}{\partial t}+(\{\boldsymbol{u}\} \cdot \nabla)\{\boldsymbol{u}\}\right)-\nabla \cdot\{\underline{\underline{\sigma}}\}=\boldsymbol{f}_{s}+\{\rho\} \boldsymbol{g}
$$

where $\boldsymbol{u}$ is the velocity, $\boldsymbol{f}_{s}$ is the surface tension force, $\boldsymbol{g}$ is the gravity, and $\underline{\underline{\boldsymbol{\sigma}}}$ is the stress tensor respecting a compressible Newtonian constitutive law:

$$
\begin{aligned}
& \underline{\underline{\boldsymbol{\sigma}}}=\underline{\underline{\boldsymbol{s}}}-p \underline{\underline{\boldsymbol{I}}} \quad \underline{\underline{\boldsymbol{s}}}=2 \mu\left(\underline{\underline{\dot{\boldsymbol{\epsilon}}}}-\frac{1}{3} \operatorname{tr}(\underline{\underline{\dot{\boldsymbol{\epsilon}}}}) \underline{\underline{\boldsymbol{I}}}\right) \\
& \operatorname{tr}(\underline{\dot{\boldsymbol{\epsilon}}})=\nabla \cdot\{\boldsymbol{u}\}
\end{aligned}
$$

where $p$ is the pressure, $\mu$ is the dynamic viscosity, $\underline{\underline{\boldsymbol{s}}}$ is the deviatoric part of $\underline{\underline{\boldsymbol{\sigma}}}, \underline{\boldsymbol{I}}$ is the identity tensor and $\underline{\underline{\dot{\boldsymbol{\epsilon}}}}$ is the strain rate tensor. Here $\nabla \cdot\{\boldsymbol{u}\}$ is a term related to the shrinkage rate deduced from the mass conservation equation as described hereafter. In the present work, we consider that surface tension appears when liquid and gas are present at the same time. This force is applied not only at the liquid/gas interface (blue curve in Figure 2a) but also at the powder/liquid boundary (white curve in Figure 2a). The former is restricted to a part of the interface $\psi=0$, while the latter boundary is immersed in the material domain. They are together detected by the contour corresponding to the presence of both liquid and gas with a liquid fraction equal to 0.5 . The surface tension can be expressed by:

$$
\boldsymbol{f}_{s}=\gamma \kappa \boldsymbol{n}
$$

where $\gamma$ is the surface tension coefficient, $\kappa=-\nabla \cdot \boldsymbol{n}$ is the average curvature and $\boldsymbol{n}$ is the unit vector normal to the interface. It should be mentioned that the tangential force resulting from the variation of $\gamma$ along the interface (Marangoni effect) is not yet taken into account in the present model. Numerical methods differ by the formulation of $\boldsymbol{\kappa} \boldsymbol{n}$. An explicit time integration method would take it at the previous time step while an implicit method computes it at the present time step, providing more stability. In fact, $\kappa \boldsymbol{n}$ can be related to the coordinate $\boldsymbol{X}$ of a point at the interface by a surface Nabla operator $\nabla_{s}[33]$ :

$$
\kappa \boldsymbol{n}=\nabla_{s} \cdot \underline{\underline{\nabla_{S} \boldsymbol{X}}}
$$

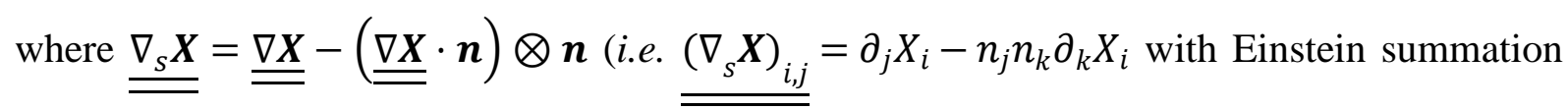
convention). We can express $\boldsymbol{X}$ by the forward Euler method:

$$
\boldsymbol{X}=\boldsymbol{X}^{-}+\boldsymbol{u} \Delta t
$$

where $\Delta t$ is the time step. Replacing $\boldsymbol{X}$ by Eq. (15), Eq. (14) becomes:

$$
\kappa \boldsymbol{n}=(\kappa \boldsymbol{n})^{-}+\nabla_{S} \cdot \underline{\underline{\nabla_{s} \boldsymbol{u}}} \Delta t
$$

It can be seen that compared with the explicit formulation, an additional term is introduced. It represents a surface diffusion of the velocity, thus introducing additional stability to the 
resolution. Following Hamide [34], such a formulation was implemented by Khalloufi et al. [35] in a semi-implicit way:

$$
\kappa \boldsymbol{n}=(\kappa \boldsymbol{n})^{-}+\left(\nabla^{2} \boldsymbol{u}-\left(\frac{\partial^{2} \boldsymbol{u}}{\partial \boldsymbol{n}^{2}}-\kappa \frac{\partial \boldsymbol{u}}{\partial \boldsymbol{n}}\right)^{-}\right) \Delta t
$$

with $/ \partial \boldsymbol{n}=\underline{\nabla \boldsymbol{u}} \cdot \boldsymbol{n}$. Here both $\boldsymbol{u}$ and $\boldsymbol{n}$ in $\partial \boldsymbol{u} / \partial \boldsymbol{n}$ and $\partial^{2} \boldsymbol{u} / \partial^{2} \boldsymbol{n}$ are taken at previous time step for the sake of consistency. However, we can also use the current unknown velocity for all terms in Eq.(16). Finally multiplying Eq.(10) by a vector test function $\boldsymbol{w}$ and combining Eqs.(11)-(13),(17) leads to the weak form which is suitable for FE implementation [33]:

$$
\begin{gathered}
\int_{\Omega} \rho \frac{\partial \boldsymbol{u}}{\partial t} \cdot \boldsymbol{w} d \Omega+\int_{\Omega} \rho(\underline{\underline{\nabla \boldsymbol{u}}} \cdot \boldsymbol{u}) \cdot \boldsymbol{w} d \Omega+2 \int_{\Omega} \mu \underline{\underline{\dot{\boldsymbol{\epsilon}}}}(\boldsymbol{u}): \underline{\underline{\dot{\boldsymbol{\epsilon}}}}(\boldsymbol{w}) d \Omega \\
-\frac{2}{3} \int_{\Omega} \mu(\nabla \cdot \boldsymbol{u})(\nabla \cdot \boldsymbol{w}) d \Omega-\int_{\Omega} p \nabla \cdot \boldsymbol{w} d \Omega \\
=\int_{\Gamma} \gamma(\kappa \boldsymbol{n})^{-} \cdot \boldsymbol{w} d \Gamma \\
+\Delta t \int_{\zeta} \gamma\left(\underline{\underline{\nabla_{s} \boldsymbol{u}}} \cdot \boldsymbol{w}\right) \cdot \boldsymbol{n}_{\zeta} d \zeta-\Delta t \int_{\Gamma} \gamma\left(\underline{\underline{\nabla_{s} \boldsymbol{u}}}: \underline{\underline{\nabla_{s} \boldsymbol{w}}}\right) d \Gamma \\
+\int_{\Omega} \rho \boldsymbol{g} \cdot \boldsymbol{w} d \Omega
\end{gathered}
$$

Here the notation $\{*\}$ is omitted for the sake of clarity. As illustrated in Figure 3 for a liquid droplet deposited onto a solid substrate, $\Gamma$ is the interface between the liquid and the gas, $\zeta$ is the boundary of $\Gamma$ with the solid substrate (contour between liquid and solid) and $\boldsymbol{n}_{\zeta}$ is the normal direction of $\zeta$ tangent to $\Gamma$. The integration by part and Gauss' law are applied to get the fourth term (arising from the compressible behavior) at left, also second and third terms at right in Eq.(18). The second term at right vanishes if we choose $\Gamma$ to be closed ( $\zeta$ vanishes). In our case, we have taken $\Gamma$ as the boundary of the whole melt pool. However, $\boldsymbol{f}_{S}$ only acts along the interface in blue and white in Figure $2 \mathrm{a}$ as $\gamma \neq 0$. As $\Gamma$ is immersed in $\Omega$, we can transform the integration over $\Gamma$ into the integration over $\Omega$ by multiplying the Dirac function associated to $\Gamma$. For the third term at right, we can show that:

$$
\begin{aligned}
\underline{\underline{\nabla_{s} \boldsymbol{u}}: \underline{\underline{\nabla_{s} \boldsymbol{w}}}} & =\left(\partial_{j} u_{i}-n_{j} n_{k} \partial_{k} u_{i}\right) \cdot\left(\partial_{j} w_{i}-n_{j} n_{l} \partial_{l} w_{i}\right) \\
& =\partial_{j} u_{i} \partial_{j} w_{i}-n_{j} n_{k} \partial_{j} w_{i} \partial_{k} u_{i} \\
= & \underline{\underline{\nabla \boldsymbol{u}}}: \underline{\underline{\nabla \boldsymbol{w}}}-(\underline{\underline{\nabla \boldsymbol{u}}} \cdot \boldsymbol{n}) \cdot(\underline{\underline{\boldsymbol{w}}} \cdot \boldsymbol{n})
\end{aligned}
$$

where $n_{i}^{2}=1$ is applied. Note that in the implementation $\boldsymbol{u}$ is taken at the current time step, while the normal direction $\boldsymbol{n}$ is taken at the previous time step. Indeed the position of the interface is not known a priori.

\subsubsection{Mass conservation}

Mass conservation is written in both the material and gas domains [36]: 


$$
\left\{\begin{array}{l}
\frac{\partial\langle\rho\rangle^{D_{1}}}{\partial t}+\nabla \cdot\langle\rho \boldsymbol{u}\rangle^{D_{1}}=0 \\
\frac{\partial\langle\rho\rangle^{D_{2}}}{\partial t}+\nabla \cdot\langle\rho \boldsymbol{u}\rangle^{D_{2}}=0
\end{array}\right.
$$

In the classical velocity-pressure formulation developed for the solution of the momentum conservation equation, the divergence of velocity, $\nabla \cdot\{\boldsymbol{u}\}$, is required. In order to deduce $\nabla \cdot\{\boldsymbol{u}\}$ from Eq. (20) we assume $\langle\rho \boldsymbol{u}\rangle^{D_{i}}=\langle\rho\rangle^{D_{i}}\langle\boldsymbol{u}\rangle^{D_{i}}$ for $i \in\{1,2\}$ and write:

$$
\nabla \cdot\langle\boldsymbol{u}\rangle^{D_{i}}=-\frac{1}{\langle\rho\rangle^{D_{i}}}\left(\frac{\partial\langle\rho\rangle^{D_{i}}}{\partial t}+\nabla\langle\rho\rangle^{D_{i}} \cdot\langle\boldsymbol{u}\rangle^{D_{i}}\right)
$$

thus leading to the following form of the global mass conservation:

$$
\begin{aligned}
\nabla \cdot\{\boldsymbol{u}\} & =\nabla \cdot\left((1-\mathcal{H})\langle\boldsymbol{u}\rangle^{D_{1}}+\mathcal{H}\langle\boldsymbol{u}\rangle^{D_{2}}\right) \\
& =(1-\mathcal{H}) \nabla \cdot\langle\boldsymbol{u}\rangle^{D_{1}}+\mathcal{H} \nabla \cdot\langle\boldsymbol{u}\rangle^{D_{2}}+\nabla \mathcal{H}\left(\langle\boldsymbol{u}\rangle^{D_{2}}-\langle\boldsymbol{u}\rangle^{D_{1}}\right) \\
& =-\frac{(1-\mathcal{H})}{\langle\rho\rangle^{D_{1}}}\left(\frac{\partial\langle\rho\rangle^{D_{1}}}{\partial t}+\nabla\langle\rho\rangle^{D_{1}} \cdot\langle\boldsymbol{u}\rangle^{D_{1}}\right)-\delta\left(\langle\boldsymbol{u}\rangle^{D_{2}}-\langle\boldsymbol{u}\rangle^{D_{1}}\right) \cdot \boldsymbol{n}_{L S}
\end{aligned}
$$

In the above relation, $\langle\rho\rangle^{D_{2}}$ is considered to be time and space independent (providing $\left.\nabla \cdot\langle\boldsymbol{u}\rangle^{D_{2}}=0\right)$ and $\nabla \mathcal{H}=-\delta \boldsymbol{n}_{L S}$ with $\boldsymbol{n}_{L S}=-\nabla \psi$ the normal direction at interface $\psi=0$ outward of gas domain. Only one velocity field is considered and some assumptions should be made to approximate the second term at right for $\psi \in[-\epsilon, \epsilon]$. A simple $1 \mathrm{D}$ case shows that a good evaluation is given by:

$$
\delta\left(\langle\boldsymbol{u}\rangle^{D_{2}}-\langle\boldsymbol{u}\rangle^{D_{1}}\right) \cdot \boldsymbol{n}_{L S}=\frac{1}{4\langle\rho\rangle^{D_{1}}} \frac{\partial\langle\rho\rangle^{D_{1}}}{\partial t} \cos ^{2}\left(\frac{\pi \psi}{2 \epsilon}\right)
$$

More details can be found in the Appendix. Finally, we can express the shrinkage term by:

$$
\dot{\theta}=\nabla \cdot\{\boldsymbol{u}\}=-\frac{(1-\mathcal{H})}{\langle\rho\rangle^{D_{1}}}\left(\frac{\partial\langle\rho\rangle^{D_{1}}}{\partial t}+\nabla\langle\rho\rangle^{D_{1}} \cdot\langle\boldsymbol{u}\rangle^{D_{1}}\right)-\frac{1}{4\langle\rho\rangle^{D_{1}}} \frac{\partial\langle\rho\rangle^{D_{1}}}{\partial t} \cos ^{2}\left(\frac{\pi \psi}{2 \epsilon}\right)
$$

\section{Numerical tests}

\subsection{Material properties}

We distinguish two zones in the material domain: the powder, $Z_{1}$, made of the solid phase $s$ and the gas phase $g$ present as porosity, and the dense matter or alumina, $Z_{2}$, made of the solid phase $s$ and the liquid phase $l$. This distinction is justified as the zones can have different properties. For instance, they have very different apparent density, thermal conductivity and viscosity. Some of these properties are directly measured or estimated for a powder bed seen as a porous medium. For other properties, such as the specific enthalpy, it is more convenient to derive properties from the individual phases. In any case, both the arrangements of phases and zones in the following verify the existence of saturated medium by: 


$$
\begin{aligned}
& \sum_{i} g_{D_{k}}^{Z_{i}}=1, \quad k \in\{1,2\} \\
& \sum_{j} g_{Z_{i}}^{\phi_{j}}=1, \quad i \in\{1,2\}
\end{aligned}
$$

The melting temperature of alumina is $2054{ }^{\circ} \mathrm{C}$ [25]. When pure alumina begins to melt or solidify, its temperature remains at $2054{ }^{\circ} \mathrm{C}$ and enthalpy increases or decreases, respectively. However, a phase transformation taking place at a constant temperature with a large enthalpy variation leads to numerical difficulties. An artificial solid-liquid transformation interval between $2004{ }^{\circ} \mathrm{C}$ ( solidus) and $2104{ }^{\circ} \mathrm{C}$ ( liquidus) is assumed, with linear relationships between the temperature and the volume fraction of the solid and liquid phases within this interval when a saturated medium with alumina is considered. Hereafter we also assume a linear one-way transformation from powder to dense matter from $1804{ }^{\circ} \mathrm{C}$ to $2104{ }^{\circ} \mathrm{C}$ shown in Figure 4, larger than the solid-liquid transformation interval. This choice comes from numerical considerations, as a rapid shrinkage from powder to dense matter leads to high velocity and numerical instability. However, the present approach is dedicated to the computation of the final bead shape which is related to liquid flow more than to shrinkage. Consequently, this choice has little influence on the final bead shape. Note that, considering a representative volume in Figure 4(b), powder in zone $Z_{1}$ may be (state 2) condensed, (state 3) partially melted and (state 6) re-solidified. Residual porosity resulting from the blocking of gas in the condensed matter (i.e., liquid or solid) is not modeled.

Material properties are given in Table 1, with gas properties approximated by the air properties. Both the density and the volumetric enthalpy in the material domain $D_{1}$ are averaged over phases:

$$
\langle\chi\rangle^{D_{1}}=\sum_{i \in\{1,2\}} \sum_{\substack{\phi_{j} \in\{s, g\} \\ \phi_{j} \in\{s, l\}(i=2)}} g_{D_{1}}^{Z_{i}} g_{Z_{i}}^{\phi_{j}} \chi^{\phi_{j}}
$$

Here $\chi$ represents the density $\rho$ and the volumetric enthalpy $\rho h$, and $g_{Z_{i}}^{\phi_{j}}$ is the volume fraction of phase $\phi_{j}$ in zone $Z_{i}$. Constant phase densities are taken. The specific enthalpy of alumina (including solid and liquid) is modeled as a polynomial function of temperature [25]. In the transformation interval, they are interpolated and weighted by their corresponding phase fraction. Figure 5(a) shows the specific enthalpy evolution of material domain $\left(D_{1}\right)$. As the mass fraction of gas is negligible in the front of alumina, the evolutions of specific enthalpy during melting and solidification are the same. Values for density and specific enthalpy are tabulated as parameters in the temperature solver.

Thermal conductivity of alumina can be found in [38] with the following expression:

$$
\lambda^{a}=5.5+34.5 e^{-0.0033 T}, T \in[25,1300]^{\circ} \mathrm{C} \text {, in } \mathrm{W} \cdot \mathrm{m}^{-1} \cdot \mathrm{K}^{-1}
$$


This expression is extrapolated to higher temperature as shown by the red curve in Figure 5(b). In order to simulate the effect of possible Marangoni flow on the homogenization of temperature in the melt pool, the thermal conductivity of liquid alumina is multiplied by an arbitrary factor 5 as initially proposed by Desmaison [22]. For the powder, several models for porous medium exist to account for the dominant value in the gas, resulting into a very low apparent thermal conductivity. The present study uses the model proposed by ZehnerSchlunder [39] with porosity fraction of 50\% (blue curve in Figure 5(b)). Finally, the thermal conductivity in the material domain $\left(D_{1}\right)$ is averaged between powder $\left(Z_{1}\right)$ and dense matter $\left(Z_{2}\right)$ :

$$
\langle\lambda\rangle^{D_{1}}=\sum_{i \in\{1,2\}} g_{D_{1}}^{Z_{i}}\langle\lambda\rangle^{Z_{i}}
$$

The rheology of the individual zones is taken as in Figure 5(c). The viscosity of the material domain is then calculated by a special law:

$$
\langle\mu\rangle^{D_{1}}= \begin{cases}\langle\mu\rangle^{Z_{1}} & \text { if } g_{D_{1}}^{Z_{1}}>0 \\ \langle\mu\rangle^{Z_{2}} & \text { otherwise }\end{cases}
$$

It means that once powder reaches the threshold temperature $\left(2104{ }^{\circ} \mathrm{C}\right)$, the powder $Z_{1}$ is fully melted $\left(g_{D_{1}}^{Z_{1}}=0\right)$ and the viscosity of the material takes the value for the dense matter $Z_{2}$. Otherwise, the viscosity of the powder always prevails, even if dense matter is partly present. In fact, if an arithmetic or geometric average viscosity between powder and dense matter would be considered for the densifying region, this would create a great increase of viscosity in this fine region, preventing liquid and powder flow (toward the melt pool). The steep transition law of Eq. (30) avoids such a viscosity peak, and provides an easy powder feeding and liquid flow. Actually, there is certainly a transient state for which the powder particles are sintered before they melt. But this transient state is so rapid that this cannot be modelled while keeping a continuum description and non infinitesimal time steps. Moreover, the viscosity of the solid part is chosen under the condition that the surface of solidified bead can be well fixed.

\subsection{Simulation configuration}

Figure 6(a) shows the whole simulation system with overall dimensions $1 \times 0.3 \times$ $1 \mathrm{~mm}^{3}$. Gas (at top, light blue, $0.1 \mathrm{~mm}$ thick) and material (below gas) are separated by a LS interface initially at height $\mathrm{Z}=0.9 \mathrm{~mm}$. A layer of powder with thickness $h_{p}$ is located between the substrate and the gas. A laser beam with radius $R_{L}=35 \mu \mathrm{m}$ scans the powder from top at velocity $v_{L}$ along the $\mathrm{X}$-direction and at mid-width, i.e., moving from position $\left(\mathrm{X}_{\mathrm{S}}, \mathrm{Y}_{\mathrm{S}}\right)=(0.2$, $0.15) \mathrm{mm}$ to position $\left(X_{E}, Y_{E}\right)=(0.8,0.15) \mathrm{mm}$. The power of the heat source is imposed progressively from $20 \%$ to $100 \%$ of effective power $P_{L}(1-R)$ from $\mathrm{X}_{\mathrm{S}}$ to $\mathrm{X}_{\mathrm{S}}+2 R_{L}$. The absorption coefficient is set to zero in gas and to different values in powder $\left(\langle\alpha\rangle^{Z_{1}}\right)$ and dense 
mater $\left(\langle\alpha\rangle^{Z_{2}}\right)$. The powder layer thickness, the effective laser power, the scanning velocity and the absorption coefficient, the viscosity and the surface tension are variables according to different cases presented hereafter, all set of values being reported in Table 2 .

The ambient temperature $20{ }^{\circ} \mathrm{C}$ is initially imposed to the entire system. In the context of SLM, such an assumption is only valid for the first passes. More complex initial temperature distributions could be envisaged in the future to account for residual heat resulting from previous passes. Convection and radiation conditions are applied at the bottom of the substrate with heat transfer coefficient $h_{T}=1000 \mathrm{~W} \cdot \mathrm{m}^{-2} \cdot \mathrm{K}^{-1}$ and emissivity $\varepsilon_{T}=0.4$. Here the value for $h_{T}$ is chosen large considering that the substrate is much smaller in the present configuration compared with that in the real process, therefore playing an important role in heat extraction. Radiation at the material/gas boundary is also taken into account as explained in Eq. (9) with $\varepsilon_{r}=0.6$. Other surfaces are adiabatic. The bottom is fixed $(\boldsymbol{u}=0)$ and velocity condition along the top is free in order to permit gas to enter into the system so as to compensate shrinkage taking place upon melting of the powder bed. Velocities normal to the four lateral vertical faces are set to zero. Time step is set to $2 \mu \mathrm{s}$.

In addition to a reference case 1, two groups of simulations are conducted, using different parameters, as detailed in Table 2. With the first group (cases 2 to 4 ) the aim is to investigate the influence of the process parameters of AM by SLM: the layer thickness $h_{p}$, the effective laser power $P_{L}(1-R)$, and the scanning velocity $v_{L}$. In the second group (cases 5 to 9 ), the effect of alumina properties is studied: liquid viscosity $\mu^{l}$, surface tension $\gamma$, material absorption coefficients $\alpha$. A total of 700,000 elements are typically used for simulations and the minimum mesh size is $0.5 \mu \mathrm{m}$ in all cases.

\section{Results and discussion}

A snapshot at $t=2 \mathrm{~ms}$ of reference case 1 (see Table 2 for detailed parameters) is shown in Figure 7. The form of the melt pool is almost stationary at this time so the unidirectional single pass process has entered a quasi-stationary regime. The mesh adaptation is illustrated in Figure 7(a). It shows a good capture of the material/gas interface and even the formation of droplets (zoomed region of (a)). The mesh adaptation technique used in order to represent the bead shape is based on error estimation as proposed by Coupez [42]. In this approach, we define the maximum number of elements $(700,000)$ and the minimum mesh size $(0.5 \mu \mathrm{m})$, together with several tracked fields, as input parameters for the calculation of an objective mesh metric, which defines for each node the aimed mesh size in different spatial directions. Mesh adaptation is based on the gradient variation of any tracked field. The greater this variation, the finer the mesh. The density field is used to refine the interfaces gas/material and powder/substrate, while the liquid fraction field is used to refine the boundary of the melt pool. Temperature and heat source are also tracked in order to get a good precision. Then the 
interpolation error of these fields is computed along the edges of the FE mesh. An optimal stretching factor of each edge can be obtained by minimizing the total interpolation errors of all mesh edges. Based on this edge stretching factor, we can construct the edge distribution tensor and the corresponding metric for each node. With a minimum mesh size of $0.5 \mu \mathrm{m}$, even small droplets (diameter around $7 \mu \mathrm{m}$ ) are well represented. The melt pool is refined, especially in regions with large gradient variation of the tracked fields, while the total number of mesh elements is kept about constant. The temperature field and the relative position between heat source and the melt pool are shown in Figure 7(b) and (c), respectively. The temperature gradient is large $\left(\sim 3 \times 10^{5} \mathrm{~K} \cdot \mathrm{mm}^{-1}\right)$ along the surface of the powder bed in front of the laser spot and in the moving direction of the heat source. Due to the weak absorption of the material, there is a deep penetration of the heat source, encompassing several times the powder layer thickness, and high temperature are also reached in depth. Behind the laser beam, heat is extracted by diffusion to the substrate and the melt pool is progressively cooled. As a consequence, the melt pool is deep just behind the heat source and it has a long and shallow tail far behind the heat source. The distribution of the volumetric heat source term arising from the Beer-Lambert law is illustrated in Figure 7(d), again revealing deep penetration into the substrate. Despite the weak absorption of ceramics, laser energy is restricted to a small region and can reach $\sim 10^{5} \mathrm{~W} \cdot \mathrm{mm}^{-3}$. When droplets are formed, they are directly exposed to the heat source and change the spatial distribution of energy. Our implementation of the heat source model adapts well when local absorption changes. One can naturally imagine that the formation of droplets is related to the value of the liquid viscosity and the surface tension. This will be discussed later.

\subsection{Influence of process parameters}

Cases 1 4 in first group are compared to investigate the influence of the process parameters. Figure 8 shows the shapes of the bead and of the melt pool at the end of the simulations for time $t=3 \mathrm{~ms}$. The skirt around the bead is due to the partial fusion and re-solidification of the powder. Comparing case 2 with case 1 , it is observed that an increase in the net laser power $(+50 \%)$ results in a larger bead, also revealed by a deeper, longer and larger melt pool. The same tendency is obtained when the scanning velocity is decreased (by $50 \%$ ) in case 3 while maintaining the laser power to the value of case 1 . These tendencies are exactly those usually observed in welding processes with the same origins [23]. Interpretation using the linear energy $\left(P_{L}(1-R) / v_{L}\right)$ is often found in the literature. It increases progressively by $50 \%$ from case 1 to case 2 , and by $33 \%$ from case 2 to case 3 . As a consequence, the melt pool is found to progressively increase from case 1 to case 3 . However, the former has a shorter tail compared to case 2. The bead shape in case 4 shows a different morphology. At the beginning of the bead formation, when the process is still unsteady, a large and high swelling appears. Although present in the previous cases, this phenomenon was 
not so pronounced. Of course it is the consequence of increasing the powder layer thickness, resulting in a larger melted volume during the unsteady regime, as well as the almost isolated region of liquid revealed by the snapshot in Figure 8. When this unsteady regime is finished, the bead retrieves a quasi-steady shape. Its depth is smaller than in case 1 despite the use of the same linear energy as melting of a thicker powder bed consumes more energy, hence not available for deeper penetration.

Temperature iso-contours of cases 1 4 are also presented in both longitudinal ( $Y=$ $0.15 \mathrm{~mm})$ and transversal sections $(X=0.8 \mathrm{~mm})$ at $t=3 \mathrm{~ms}$ in Figure 9. For all cases, the temperature gradient in front of the laser is much higher than behind it, especially in the powder bed, which has a lower thermal conductivity. The temperature iso-contours of cases 2 and 3 are similar, both with a deeper penetration compared with case 1 . However, case 3 has a smaller temperature gradient at the tail of melt pool as the laser moves more slowly. Comparing case 4 with 1 confirms previous observations: more energy is absorbed by the powder bed as the layer thickness increases. As a result, the melt pool in substrate is smaller than that of case 1 .

\subsection{Influence of material parameters}

\subsubsection{Effect of absorption coefficient}

The influence of the absorption coefficient is significant as it can totally change the energy distribution. The melt pool of case 5 (Figure 10(a)) is much smaller than that of Case 1 when the local absorption is decreased (25\% in powder and $33 \%$ in substrate). This melt pool is limited to the upper part of the substrate. A decrease of the local absorption will melt only the powder layer. On the other hand, if we had to consider an extreme case with infinite absorption of powder, laser energy would be totally absorbed on the powder surface. In this situation, heat transfer into the substrate would only develop by conduction. Once again, it would be hard to well penetrate into the substrate. Consequently, the local absorption should be well adapted in order to keep a suitable penetration depth and thus good bonding between consolidated layers. Although the absorption is lower in case 5, high temperature can be also reached in almost the same depth in the substrate, comparing the iso-temperature $T=900{ }^{\circ} \mathrm{C}$ with that of case 1 . The profile of the heat source term along the vertical laser beam direction is plotted in Figure 11 in order to help explaining this phenomenon.

Figure 11 is plotted at the beginning of the simulation in order to show the heat source distribution in full layer thickness. At this moment, the distance travelled by the heat source is only $4 \mu \mathrm{m}$, i.e. mush smaller than $70 \mu \mathrm{m}$ required to reach nominal power of the heat source. In case 1 , the theoretical value of $\dot{q}_{L}$ at the surface of the powder bed $(Z=0.9 \mathrm{~mm})$ is $4.1 \times 10^{4} \mathrm{~W} \cdot \mathrm{mm}^{-3}$ according to Eq. (8). Figure $11 \mathrm{~b}$ shows a smaller value. This difference comes from the homogenization of the absorption coefficient between the two domains 
around $\psi=0(Z=0.9 \mathrm{~mm})$. Due to this homogenization, the local absorption in $\psi \in[0, \epsilon]$ is not zero, and thus a small part of laser energy is absorbed. On the other hand, local absorption in $\psi \in[-\epsilon, 0]$ becomes smaller due to the averaging between powder and gas. These two reasons result into the principal difference between theoretical and simulated value of $\dot{q}_{L}$, together with the loss of precision due to numerical implementation. Nevertheless, this difference is small and it can be decreased with a smaller thickness $(\epsilon)$ of the transition zone. In the powder bed, the heat source term decreases exponentially due to local energy absorption. The change of absorption coefficient from powder $\left(\langle\alpha\rangle^{Z_{1}}=4 \mathrm{~mm}^{-1}\right)$ to dense matter $\left(\langle\alpha\rangle^{Z_{2}}=3 \mathrm{~mm}^{-1}\right)$ results in a steep change of $\dot{q}_{L}$ at the powder/substrate interface $(Z=0.87 \mathrm{~mm})$. The reason of this steep change can be found in Eq.(8) where the $\alpha$ coefficient appears as a multiplier in the expression of the source term. Below the interface, the source term decreases again exponentially until truncation depth at $Z=0.05 \mathrm{~mm}$. Comparing case 5 with case 1 , even though the absorption of case 5 is lower than that of case 1 , the heat source in case 5 is more powerful for $Z<0.5 \mathrm{~mm}$. The reason is that the attenuation of case 5 is lower than that of case 1, leading to an intersection of the two curves. Considering the sensibility of temperature field to absorption, this parameter thus needs good calibration with experiments.

\subsubsection{Effect of viscosity and surface tension}

The final bead shape is the result of the dynamics of the melt pool and its solidification. The melt pool/gas interface is mostly affected by liquid viscosity and surface tension. Figure 12 shows transversal cross sections of cases $1,6,7,8$ and 9 after reaching a stable shape for the melt pool. The effect of increasing viscosity is accessible by comparing cases 1,6 , and 7 . The surface tension becomes insufficient to drive the lateral melt pool downward up to the substrate prior to solidification. In addition, since the two lateral sides keep wetting the powder, more powder is melted by conduction in cases 6 and 7. On the other hand, the effect of decreasing surface tension is shown with cases 1,8 and 9. The same global effect is found on the transverse cross section of the bead. Although the liquid does not wet the powder at this moment in case 8 , it has a larger bead because the surface tension is decreased and powder was previously wetted by liquid during a longer period. The extreme case 9 without effect of surface tension is also presented. As the gravity is less significant than the surface tension, melt pool keeps wetting the powder and it is solidified rapidly before all liquid can reach the same horizontal level. Consequently, the final bead is in convex shape.

The effect of surface tension is so significant that droplets can be formed as mentioned above, requiring a good mesh adaptation to capture this effect. To investigate this phenomenon, 6 snapshots covering a $0.12 \mathrm{~ms}$ duration sequence are presented in Figure 13 for case 1. When there is a gap between the liquid pool and the powder bed in the spot of the laser, an abrupt cliff of powder forms. It is heated and melted (1.66 ms). The melted zone is then spheroidized under the effect of surface tension and several small droplets start forming 
(1.68 ms). When more powder is melted, the droplets may coalesce together $(1.7 \mathrm{~ms})$ to form bigger droplets $(1.74 \mathrm{~ms})$. One or several such droplets fall down into the melt pool $(1.76 \mathrm{~ms})$. It results into a liquid wave towards the tail of melt pool and again a steep cliff between the powder bed surface and the melt pool $(1.78 \mathrm{~ms})$. This may lead to wrinkles on the surface of the solidified bead when the wave arrives to the tail of the melt pool where final solidification occurs. As shown in this sequence of snapshots, there is a kind of periodicity which can be observed in this phenomenon. It should also be underlined that the fall of droplets due to gravity and the spheroidization due to surface tension generate high velocities (in the range of $0 \sim 0.5 \mathrm{~m} \cdot \mathrm{s}^{-1}$ in this example). It can be seen that the developed solver with semi-implicit formulation of surface tension demonstrates its robustness, delivering stable results to reproduce this dynamics. Unlike case 1, no formation of droplets is observed in case 8 and 9 and the melt pool is always stable as shown in Figure 13. The front of the liquid pool keeps wetting the powder and the melt pool keeps moving forward continuously with a velocity close to the scanning velocity.

\subsection{Multiple passes with different scanning strategies}

At last, two simulations of 3 passes with the parameters of the reference case 1 but different scanning strategies are presented. As shown in Figure 14, the whole system is enlarged to $1 \times 0.5 \times 1 \mathrm{~mm}^{3}$. Both multi-pass simulations have a hatch distance $2 R_{L}=$ $70 \mu \mathrm{m}$ and 800,000 elements are used. Temperature is recorded at 9 positions located in the substrate just below the powder layer. Strategies with (case A) unidirectional scanning and (case B) alternate scanning are compared in Figure 14 and Figure 15. In order to preserve the bead shape, mesh elements around the solidified bead surface are fixed [43] during remesh procedure.

In Figure 14, temperature field and melt pool shape are displayed on the material surface using a sequence of successive snapshots. Generally, the melt pool of both cases becomes larger from the first to the last pass since the whole system is progressively heated with time. During the first pass, both strategies are the same so only one snapshot is shown at $t=$ $1.48 \mathrm{~ms}$. Result is very close to the reference case 1 . When the laser starts the second pass $(t=3.2 \mathrm{~ms})$ in case A, the beginning of the first pass is already cooled. The laser thus heats a region close to the ambient temperature. However, in case $\mathrm{B}$, as a U-turn takes place between successive passes, the turn back zone is heated during a longer time. Thus, high temperature can be reached and bigger melt pool is obtained than with unidirectional scanning. This is shown at $t=4.48 \mathrm{~ms}$. This even leads to two isolated melt pools (case B) at $t=5.6 \mathrm{~ms}$ as the tail of the melt pool is still in a hot region and requires a larger time to solidify. At the end of the last pass, the maximum temperature is around $2550{ }^{\circ} \mathrm{C}$ in both cases $\mathrm{A}$ and $\mathrm{B}$. This maximum temperature is achieved on the surface exposed in the laser spot and it depends mainly on laser power and velocity and also the absorption. One significant difference comes 
from the bead shape. In unidirectional scanning (case A), the first bead is not remelted while the second is. The last bead becomes larger when it remelts the second. In alternate scanning (case B), as there is a turn between passes, the end of the first and second bead are remelted and connected

Figure 15 shows the temperature evolution at sampling positions given in Figure 14. All positions are heated rapidly, with typical heating rate $10^{7}{ }^{\circ} \mathrm{C} \cdot \mathrm{s}^{-1}$, to almost the same maximum temperature (around $2500{ }^{\circ} \mathrm{C}$ ) when exposed to the laser beam. Once leaving the laser spot, they cool down with an average cooling rate of $7.5 \times 10^{5}{ }^{\circ} \mathrm{C} \cdot \mathrm{s}^{-1}$. The cooling rate in the artificial enlarged interval $T \in[2004,2104]{ }^{\circ} \mathrm{C}$ is much lower due to the release of latent heat (Figure 5(a)). Position 1 in case B is cooled during a longer duration than that in case A, since the laser trajectory approaches it only at the end of the second pass in case B while at the beginning in case $\mathrm{A}$. In case $\mathrm{B}$, position 3 is passed beside by laser after a short duration of $t \approx 3.5 \mathrm{~ms}$ and then slightly heated, while this happens at $t \approx 5.5 \mathrm{~ms}$ in case $\mathrm{A}$. However, none of them is remelted as the hatch distance is too large to cover again the same location. Position 6 in case B experiences a higher temperature as it is preheated by conduction when the laser reaches the end of the first pass. During the third pass, the whole system is warmer than at the beginning, so higher maximum temperature and slower cooling can be observed (positions 7, 8 and 9). In conclusion, the temperature history can be predicted as a function of the scanning strategies and could obviously be linked to process parameters such as the hatch distance, the laser velocity and power, thus providing with a mean to control the temperature history and the solidification paths, and consequently the final structures.

\section{Conclusions}

AM by SLM applied to a ceramic material is modeled by a Level Set FE method at the scale of bead formation. A volume heat source is proposed considering the local absorption of the laser-transparent material. The powder bed is considered as a continuum and its apparent volume shrinkage upon melting is modeled by a compressible Newtonian law. A semiimplicit formulation for the implementation of the surface tension provides a stable and efficient numerical solution of the momentum conservation. The bead shape is tracked by a level set method and dynamic mesh adaptation is used to capture the fusion of the powder bed into droplets, their gathering into the fusion zone, and the continuous evolution of the geometry of the bead as a result of viscosity and surface tension. It is noteworthy to mention that many of these developments are not limited to ceramics and can be further developed to be used for other materials.

Several unidirectional single pass cases are compared to investigate the influence of both the process parameters and the material properties on the temperature distribution and bead shape. It can be emphasized that for such transparent ceramic material, the absorption 
coefficient is a very important parameter to calibrate, which has a large influence on temperature evolution and melt pool shape. The final bead shape is well related to liquid viscosity and surface tension and expected evolutions are retrieved when varying these parameters. Two 3-pass simulations are also presented, with which the importance of the scanning strategy on the temperature evolution inside the material is demonstrated.

Future work is required for modeling of solidification structures and prediction of the deformation of the solid material to investigate the influence of process parameters on final structures and stress distribution. Multi-layer simulations are also to be conducted. Moreover experimental observations should be developed in order to validate the bead geometry and liquid pool shape. These observations will help to estimate unknowns or badly estimated parameters in a calibration approach.

\section{Acknowledgements}

This work has been conducted within the framework of the CEFALE project, part of the ACLAME program funded by the Institute CARNOT MINES (Paris, FR). The authors would like to thank Christophe Colin, Jean-Dominique Bartout, Marie-Hélène Berger and Liliana Moniz Da Silva Sancho from the MINES ParisTech Centre des Matériaux (Evry, FR) for invaluable information regarding AM by SLM and the ceramic material.

\section{Appendix}

A simple 1D case is proposed for the estimation of the term $\nabla \mathcal{H}\left(\langle\boldsymbol{u}\rangle^{D_{2}}-\langle\boldsymbol{u}\rangle^{D_{1}}\right)$ where materials are compacted in direction $-Z$ (as $\psi$ and $Z$ are in the same direction). The magnitude of the velocity is found to increase linearly in $[-\epsilon, 0]$ and remains constant in $[0, \epsilon]$. This leads to write Eq. (20) as:

$$
\left\{\begin{array}{c}
\nabla \cdot\langle\boldsymbol{u}\rangle^{D_{1}}=\frac{\partial\left\langle u_{Z}\right\rangle^{D_{1}}}{\partial Z}=\frac{-1}{\langle\rho\rangle^{D_{1}}}\left(\frac{\partial\langle\rho\rangle^{D_{1}}}{\partial t}+\nabla\langle\rho\rangle^{D_{1}} \cdot\langle\boldsymbol{u}\rangle^{D_{1}}\right) \approx \frac{-1}{\langle\rho\rangle^{D_{1}}} \frac{\partial\langle\rho\rangle^{D_{1}}}{\partial t} \\
\nabla \cdot\langle\boldsymbol{u}\rangle^{D_{2}}=\frac{\partial\left\langle u_{Z}\right\rangle^{D_{2}}}{\partial Z}=\frac{-1}{\langle\rho\rangle^{D_{2}}}\left(\frac{\partial\langle\rho\rangle^{D_{2}}}{\partial t}-\nabla\langle\rho\rangle^{D_{2}} \cdot\langle\boldsymbol{u}\rangle^{D_{2}}\right)=0
\end{array}\right.
$$

The approximation of the first equality in the expression of gradient of $\nabla\langle\rho\rangle^{D_{1}}$ is due to the negligible spatial variation of $\langle\rho\rangle^{D_{1}}$ in front of its temporal variation. In the second expression, the previous hypothesis of a fixed gas density is used.

The constant velocity of the gas domain in the transition zone $[-\epsilon, \epsilon],\left\langle u_{0}\right\rangle^{D_{2}}$, is considered as equal to the material velocity, $\left\langle u_{Z}\right\rangle^{D_{1}}$, at material/gas interface for continuity reasons. Thus the two averaged velocities can be expressed by:

$$
\left\{\begin{array}{c}
\left\langle u_{Z}\right\rangle^{D_{1}}=\left\langle u_{0}\right\rangle^{D_{2}}-\frac{\psi}{\langle\rho\rangle^{D_{1}}} \frac{\partial\langle\rho\rangle^{D_{1}}}{\partial t} \\
\left\langle u_{Z}\right\rangle^{D_{1}}=\left\langle u_{0}\right\rangle^{D_{2}} \quad \psi \in[0, \epsilon]
\end{array} \quad \psi \in[-\epsilon, 0]\right.
$$


Then we average the velocity difference in the transition zone $\psi \in[-\epsilon, \epsilon]$ in order to estimate the second term in Eq.(22):

$$
\left(\langle\boldsymbol{u}\rangle^{D_{2}}-\langle\boldsymbol{u}\rangle^{D_{1}}\right) \cdot \boldsymbol{n} \approx \frac{1}{2 \epsilon} \int_{\epsilon}^{\epsilon}\left(\left\langle u_{Z}\right\rangle^{D_{2}}-\left\langle u_{Z}\right\rangle^{D_{1}}\right) d \psi=\frac{1}{4\langle\rho\rangle^{D_{1}}} \frac{\partial\langle\rho\rangle^{D_{1}}}{\partial t} \epsilon
$$

Finally, with $\delta$ function this term is equal to:

$$
\delta\left(\langle\boldsymbol{u}\rangle^{D_{2}}-\langle\boldsymbol{u}\rangle^{D_{1}}\right) \cdot \boldsymbol{n}=\frac{1}{4\langle\rho\rangle^{D_{1}}} \frac{\partial\langle\rho\rangle^{D_{1}}}{\partial t} \cos ^{2}\left(\frac{\pi \psi}{2 \epsilon}\right)
$$

1D test shows that this proposition improves the mass conservation of the whole system during simulation. Although it is based on a 1D simple case and several assumptions have been made, the same expression is introduced in 3D simulations. 


\section{References}

[1] E. O. Olakanmi, R. F. Cochrane and K. W. Dalgarno, A review on selective laser sintering/melting (SLS/SLM) of aluminium alloy powders: Processing, microstructure, and properties, Prog. Mater. Sci. 74 (2015) 401-477.

[2] M. Akita, Y. Uematsu, T. Kakiuchi, M. Nakajima and R. Kawaguchi, Defect-dominated fatigue behavior in type 630 stainless steel fabricated by selective laser melting, Mater. Sci. Eng. A 666 (2016) 19-26.

[3] Y. J. Liu, S. J. Li, H. L. Wang, W. T. Hou, Y. L. Hao, R. Yang, T. B. Sercombe and L. C. Zhang, Microstructure, defects and mechanical behavior of beta-type titanium porous structures manufactured by electron beam melting and selective laser melting, Acta Mater. 113 (2016) 56-67.

[4] H. Schwab, F. Palm, U. Kühn, and J. Eckert, Microstructure and mechanical properties of the near-beta titanium alloy Ti-5553 processed by selective laser melting, Mater. Des. 105 (2016) 75-80.

[5] M. Averyanova, P. Bertrand and B. Verquin, Manufacture of Co-Cr dental crowns and bridges by selective laser Melting technology, Virtual Phys. Prototyp. 6 (2011) 179-185.

[6] N. J. Harrison, I. Todd and K. Mumtaz, Reduction of micro-cracking in nickel supperalloys processed by Selective Laser Melting: A fundamental alloy design approach, Acta Mater. 94 (2015) 59-68.

[7] http://www.custompartnet.com/wu/selective-laser-sintering, 2016.

[8] C. Y. Yap, C. K. Chua, Z. L. Dong, Z. H. Liu, D. Q. Zhang, L. E. Loh and S. L. Sing, Review of selective laser melting: Materials and applications, Appl. Phys. Rev. 2 (2015) 041101.

[9] J.-P. Kruth, G. Levy, F. Klocke and T. H. C. Childs, Consolidation phenomena in laser and powder-bed based layered manufacturing, CIRP Ann. - Manuf. Technol. 56 (2007) 730-759.

[10] Y.-C. Hagedorn, J. Wilkes, W. Meiners, K. Wissenbach and R. Poprawe, Net shaped high performance oxide ceramic parts by selective laser melting, Phys. Procedia 5 (2010) 587-594.

[11] J. F. Li, L. Li and F. H. Stott, Comparison of volumetric and surface heating sources in the modeling of laser melting of ceramic materials, Int. J. Heat Mass Transf. 47 (2004) 1159-1174.

[12] N. E. Hodge, R. M. Ferencz and J. M. Solberg, Implementation of a Thermomechanical Model for the Simulation of Selective Laser Melting, Comput. Mech. 54 (2014) 33-51.

[13] A. V. Gusarov, I. Yadroitsev, P. Bertrand and I. Smurov, Model of Radiation and Heat Transfer in Laser-Powder Interaction Zone at Selective Laser Melting, J. Heat Transf. 131 (2009) 72-101. 
[14] W. King, A. T. Anderson, R. M. Ferencz, N. E. Hodge, C. Kamath and S. A. Khairallah, Overview of modelling and simulation of metal powder bed fusion process at Lawrence Livermore National Laboratory, Mater. Sci. Technol. 31 (2015) 957-968.

[15] Y. Li and D. Gu, Thermal behavior during selective laser melting of commercially pure titanium powder: Numerical simulation and experimental study, Addit. Manuf. 1-4 (2014) 99-109.

[16] I. A. Roberts, C. J. Wang, R. Esterlein, M. Stanford and D. J. Mynors, A threedimensional finite element analysis of the temperature field during laser melting of metal powders in additive layer manufacturing, Int. J. Mach. Tools Manuf. 49 (2009) 16-923.

[17] J. P. Kruth, X. Wang, T. Laoui and L. Froyen, Lasers and materials in selective laser sintering, Assem. Autom. 23 (2003) 57-371.

[18] G. Marion, G. Cailletaud and C. Colin, A finite element model for the simulation of direct metal deposition, presented at the ICALEO, San Diego, USA, 2014.

[19] C. Körner, E. Attar and P. Heinl, Mesoscopic simulation of selective beam melting processes, J. Mater. Process. Technol. 211 (2011) 978-987.

[20] J. Zhou, Y. Zhang and J. K. Chen, Numerical simulation of laser irradiation to a randomly packed bimodal powder bed, Int. J. Heat Mass Transf. 52 (2009) 3137-3146.

[21] S. Osher and J. A. Sethian, Fronts propagating with curvature-dependent speed: Algorithms based on Hamilton-Jacobi formulations, J. Comput. Phys. 79 (1988) 12-49.

[22] O. Desmaison, M. Bellet and G. Guillemot, A level set approach for the simulation of the multipass hybrid laser/GMA welding process, Comput. Mater. Sci. 91 (2014) 240250.

[23] S. Chen, G. Guillemot and Ch.-A. Gandin, Three-dimensional cellular automaton-finite element modeling of solidification grain structures for arc-welding processes, Acta Mater. 115 (2016) 448-467.

[24] M. Shakoor, B. Scholtes, P.-O. Bouchard and M. Bernacki, An efficient and parallel level set reinitialization method - Application to micromechanics and microstructural evolutions, Appl. Math. Model. 39 (2015) 7291-7302.

[25] M. W. Chase, Thermochemical tables. NIST-JANAF, 1998.

[26] A. Saad, Ch.-A. Gandin and M. Bellet, Temperature-based energy solver coupled with tabulated thermodynamic properties - Application to the prediction of macrosegregation in multicomponent alloys, Comput. Mater. Sci. 99 (2015) 221-231.

[27] J. Lawrence, An analysis of the beam interaction characteristics of selected lasers with an alpha-alumina bioceramics, Optics \& Lasers Eng., 41 (2004) 505-514.

[28] H. Gong, K. Rafi, T. Starr and B. Stucker, Effect of defects on fatigue tests of as-built Ti-6Al-4V parts fabricated by selective laser melting, presented at the Solid Freeform Fabrication Symposium, 499-506, Austin Texas, 2012. 
[29] G. Defillon, J. Gueusquin and N. Iooss, Modèle du bain de fusion de SLM sur céramique avec disparition de l'agent absorbant restreinte au bain, Internal report, Ecole des Mines ParisTech, 2014.

[30] J. U. Brackbill, D. B. Kothe and C. Zemach, A continuum method for modeling surface tension, J. Comput. Phys. 100 (1992) 335-354.

[31] S. S. Sih and J. W. Barlow, The prediction of the emissivity and thermal conductivity of powder beds, Parti. Sci. \& Tech. 22 (2010) 427-440.

[32] E. Louvis, P. Fox and C. J. Sutcliffe, Selective laser melting of aluminium components, J. Mater. Process. Technol. 211 (2011) 275-284.

[33] S. Hysing, A new implicit surface tension implementation for interfacial flows Modeling of Surface Tension Effects, Int. J. Num. Meth. Flu. 51 (2005) 659-672.

[34] M. Hamide, Modélisation numérique du soudage à l'arc des aciers, $\mathrm{PhD}$ thesis, MinesParisTech, 2008.

[35] M. Khalloufi, Y. Mesri, R. Valette, E. Massoni and E. Hachem, High fidelity anisotropic adaptive variational multiscale method for multiphase flows with surface tension, Comput. Methods Appl. Mech. Eng. 307 (2016) 44-67.

[36] M. Rappaz, M. Bellet and M. Deville, Numerical modeling in materials science and engineering, first ed., Springer, Lausanne, 2003.

[37] R. Morrell, Handbook of Properties of Technical \& Engeering Ceramics. H.M.S.O., London, 1985.

[38] Y. S. Touloukian, R. K. Kirby, R. E. Taylor and T. T. R. Lee, Thermal expansion nonmetallic solids, Thermophys. Prop. Matter. 13 (1984) 176-177.

[39] P. Zehner and E. U. Schlunder, Thermal conductivity of granular materials at moderate temperature (in German), Chem. Ing. Tech. 42 (1970) 933-941.

[40] Y. Kawai and Y. Shiraishi, Handbook of Physico-chemical Properties at High Temperatures, ISIJ, 1988.

[41] J. M. Lihrmann and J. S. Haggerty, Surface Tensions of Alumina-Containing Liquids, J. Am. Ceram. Soc. 68 (1985) 81-85.

[42] T. Coupez, Metric construction by length distribution tensor and edge based error for anisotropic adaptive meshing, J. Comput. Phys. 230 (2011) 2391-2405.

[43] M. Shakoor, P.-O. Bouchard and M. Bernacki, An adaptive level-set method with enhanced volume conservation for simulations in multiphase domains, Int. J. Num. Meth. Eng. (2016) 1097-0207. 


\section{List of tables:}

Table 1: Material properties for pure alumina.

Table 2: Parameters for the different simulation cases. Red color highlights the parameters changed with respect to the reference case.

\section{List of figures:}

Figure 1: Schematics of additive manufacturing (AM) by selective laser melting (SLM) [7].

Figure 2: Schematics of (a) modeling of additive manufacturing (AM) by selective laser melting (SLM) with the level set (LS) method used to track the isovalue $\psi=0$ of the signed distance function to the interface and (b) numerical implementation of the heat source (FE mesh in black, regular grid in blue).

Figure 3: Surface, interface and normal vectors defined for the integration of the surface tension term imposed at the liquid/gas interface.

Figure 4: One-way transformation from powder (zone $Z_{1}$ ) to dense matter (zone $Z_{2}$ ) with (a) volume fractions as a function of temperature in domain $D_{1}$ and (b) illustration of possible distribution of zones and phases depending on the maximum heating temperature reached during the thermal history, (state 5) above and (state 6) below the melting temperature of the dense matter. Other states represent intermediate status of domain $D_{1}$ during the transformation path.

Figure 5: Temperature evolution of (a) the specific enthalpy in the whole domain $D_{1}$, (b) the thermal conductivity and (c) the dynamic viscosity of $\left(Z_{1}\right)$ powder and $\left(Z_{2}\right)$ dense matter.

Figure 6: Configuration of unidirectional single pass simulation with overall dimensions, $(\mathrm{O}$, $\mathrm{X}, \mathrm{Y}, \mathrm{Z}$ ) frame and direction of the scanning velocity. Gas is with transparent rendering so as to reveal the initial position of the laser in a cut view at mid-width of the powder plus substrate sub-domains showing a multicolor temperature field.

Figure 7: Snapshot for reference case at $t=2 \mathrm{~ms}$ illustrating (a) the FE mesh adaptation in the vicinity of the pool surface and $(a, b)$ temperature field (cut view along the vertical symmetry plane), (c) the melt pool defined by regions of temperature higher or equal to the melting temperature in the material domain (transparent rendering is active in order to show the whole melt pool), and (c, d) position of the heat source and distribution of the volumetric heat source term (cut view along the vertical symmetry plane). Gas is removed and the black line shows the powder/substrate boundary.

Figure 8: Simulated bead shape (center) and melt pool shape (two sides) for cases 1 4 at $t=3 \mathrm{~ms}$. Gas and powder are removed and the red contour indicates the melt pool. The skirt 
around the bead corresponds to powder partially melted and solidified. The black line is the powder/substrate boundary.

Figure 9: Longitudinal $(Y=0.15 \mathrm{~mm})$ and transversal $(X=0.6 \mathrm{~mm})$ section views of cases $1-4$ at $t=3 \mathrm{~ms}$. Black curves are temperature iso-contours in the range $\left[900{ }^{\circ} \mathrm{C}, 2100{ }^{\circ} \mathrm{C}\right.$ ] with $200^{\circ} \mathrm{C}$ temperature steps, thus below the melting temperature, permitting a clear comparison of the melt pool depths. The white horizontal line is the powder/substrate boundary. The white vertical line in the longitudinal section indicates the position of the transversal section.

Figure 10: Displays from the analysis of the case 5 simulation showing (a) the shape of the bead and the melt pool at $t=3 \mathrm{~ms}$ with the same scale as in Figure 8, and (b) the heat source distribution and temperature iso-contours with the same scale as in Figure 9.

Figure 11: (a) Heat source distribution and (b) profile along laser axe of case 1 (red) and 5 (black) with different absorption coefficient at $t=4 \mu$ s (corresponding to $24.6 \%$ of $P_{L}(1-$ $R$ ) imposed).

Figure 12: Transversal cross sections at $X=0.5 \mathrm{~mm}, t=2 \mathrm{~ms}$ of case 1, 6, 7, 8 and 9. Gas and powder are removed.

Figure 13: Comparison of melt pool of cases 1,8 and 9 with different value of the surface tension. The melt pool (red, iso-contour of liquidus) and the laser spot (black contour) with $R_{L}=35 \mu \mathrm{m}$ are highlighted. The surface in cases 8 and 9 is drawn with transparency in order to better show the velocity field as the fluid flows is principally developed below the surface.

Figure 14: Temperature field at different times during 3 passes with (\#A) unidirectional scanning and (\#B) alternate scanning. The black contour is the melt pool. Temperature range is scaled in $400 \sim 2700{ }^{\circ} \mathrm{C}$ in order to have a good contrast.

Figure 15: Temperature evolution of local points 1 9 for (\#A) unidirectional scanning and (\#B) alternate scanning. 


\begin{tabular}{|c|c|c|c|c|}
\hline Properties & Symbol & Value & Unit & Ref. \\
\hline solid, liquid, gas & $s, l, g$ & & & \\
\hline Powder zone & $Z_{1}$ & $s, g$ & & \\
\hline Dense matter zone & $Z_{2}$ & $s, l$ & & \\
\hline $\begin{array}{l}\text { Volume fraction of phase } \phi_{j} \text { in } \\
\text { zone } Z_{i}\end{array}$ & $g_{Z_{i}}^{\phi_{j}}$ & & & \\
\hline $\begin{array}{l}\text { Volume fraction of zone } Z_{i} \text { in } \\
\text { material domain } D_{1}\end{array}$ & $g_{D_{1}}^{Z_{i}}$ & & & \\
\hline Solid and liquid alumina density & $\rho^{S}, \rho^{l}$ & 3800 & $\mathrm{~kg} \cdot \mathrm{m}^{-3}$ & [37] \\
\hline Gas density & $\rho^{g}$ & 1.3 & $\mathrm{~kg} \cdot \mathrm{m}^{-3}$ & \\
\hline $\begin{array}{l}\text { Enthalpy per unit mass of } \\
\text { material domain }\left(D_{1}\right)\end{array}$ & $\langle h\rangle^{D_{1}}$ & Figure 5(a) & $\mathrm{J} \cdot \mathrm{kg}^{-1}$ & [25] \\
\hline Heat capacity of gas & $C_{p}^{g}$ & 1000 & $\mathrm{~J} \cdot \mathrm{kg}^{-1} \cdot \mathrm{K}^{-1}$ & \\
\hline Alumina conductivity & $\lambda^{a}$ & Eq.(28) & $\mathrm{W} \cdot \mathrm{m}^{-1} \cdot \mathrm{K}^{-1}$ & [38] \\
\hline Gas conductivity & $\lambda^{g}$ & 0.024 & $\mathrm{~W} \cdot \mathrm{m}^{-1} \cdot \mathrm{K}^{-1}$ & \\
\hline Conductivity of zone $i$ & $\langle\lambda\rangle^{Z_{i}}$ & Figure 5(b) & $\mathrm{W} \cdot \mathrm{m}^{-1} \cdot \mathrm{K}^{-1}$ & [39] \\
\hline Viscosity of liquid alumina & $\mu^{l}$ & 0.069 & $\mathrm{~Pa} \cdot \mathrm{s}$ & [40] \\
\hline Viscosity of gas & $\mu^{g}$ & 0.00024 & $\mathrm{~Pa} \cdot \mathrm{s}$ & \\
\hline Viscosity of zone $i$ & $\langle\mu\rangle^{Z_{i}}$ & Figure 5(c) & $\mathrm{Pa} \cdot \mathrm{s}$ & \\
\hline Surface tension liquid/gas & $\gamma$ & 0.067 & $\mathrm{~N} \cdot \mathrm{m}^{-1}$ & [41] \\
\hline
\end{tabular}

Table 1: Material properties for pure alumina. 


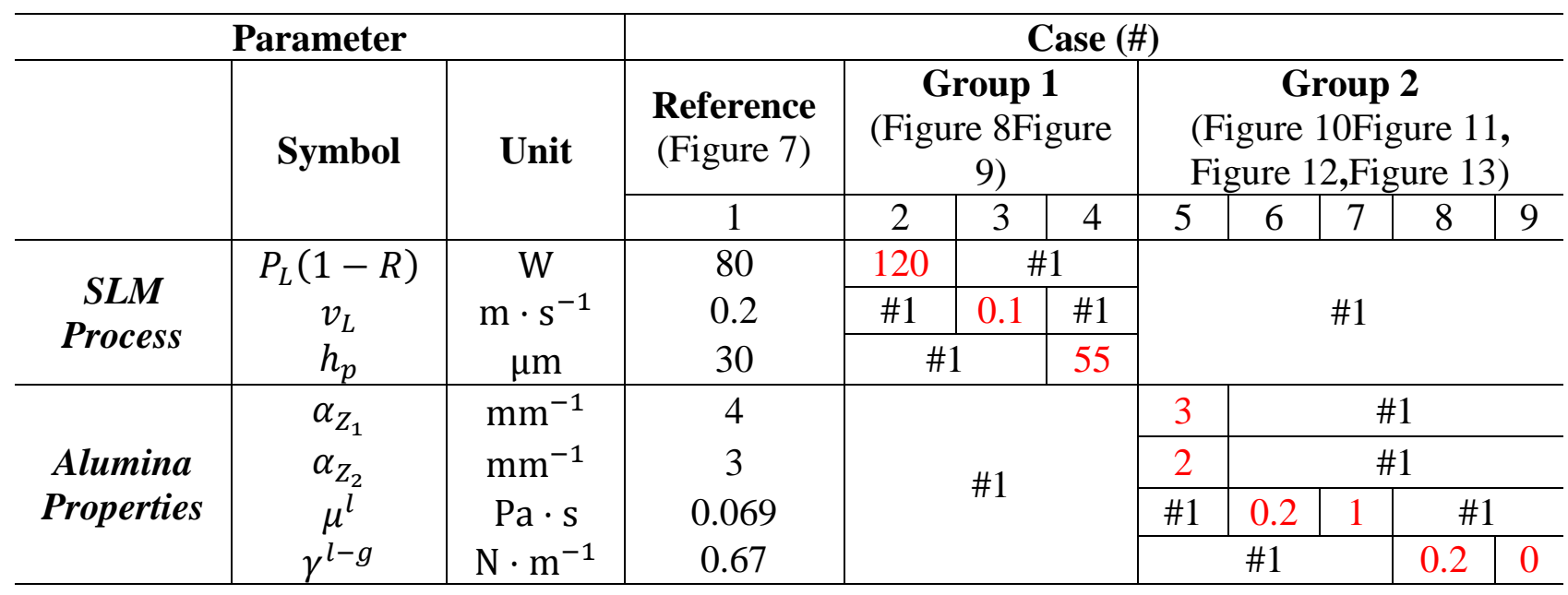

Table 2: Parameters for the different simulation cases. Red color highlights the parameters changed with respect to the reference case. 


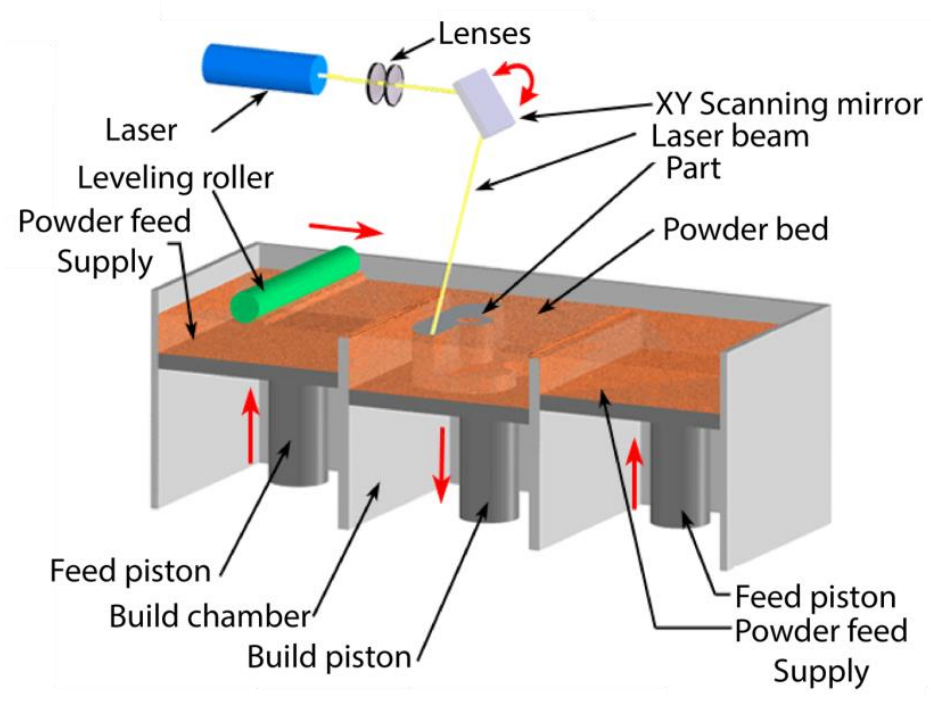

Figure 1: Schematics of additive manufacturing (AM) by selective laser melting (SLM) [7]. 

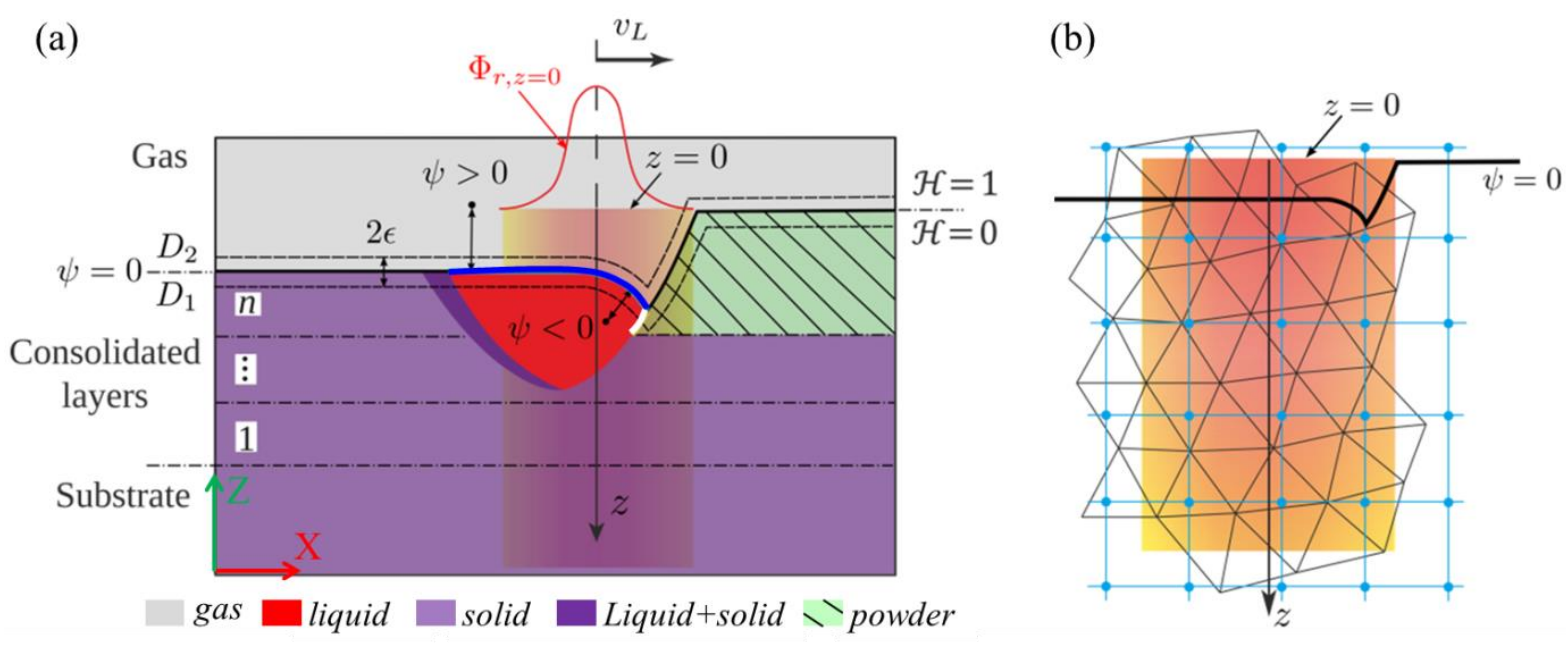

Figure 2: Schematics of (a) modeling of additive manufacturing (AM) by selective laser melting (SLM) with the level set (LS) method used to track the isovalue $\psi=0$ of the signed distance function to the interface and (b) numerical implementation of the heat source (FE mesh in black, regular grid in blue). 


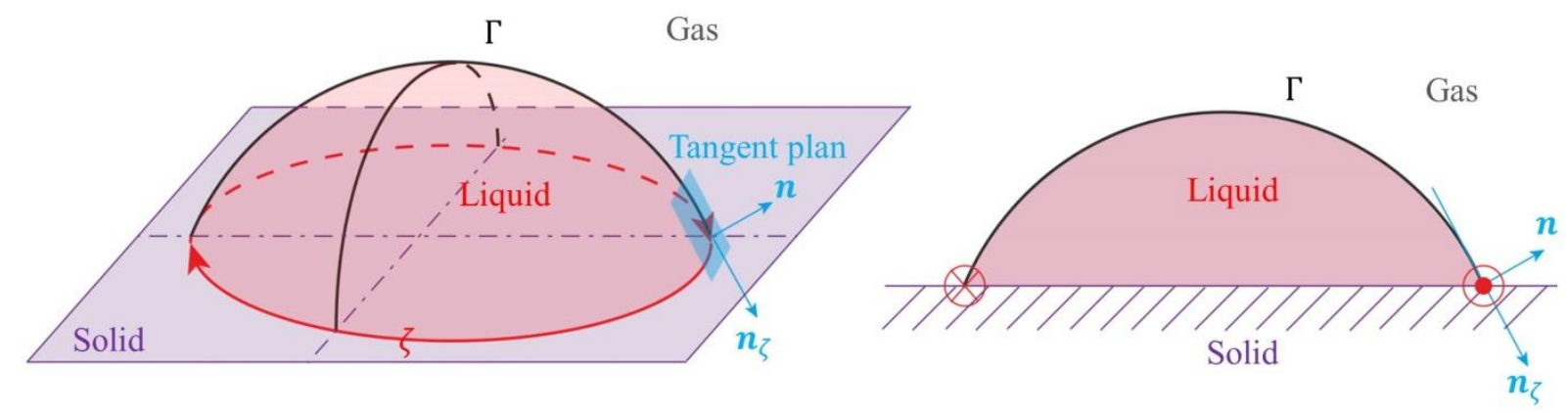

Figure 3: Surface, interface and normal vectors defined for the integration of the surface tension term imposed at the liquid/gas interface. 
(a)

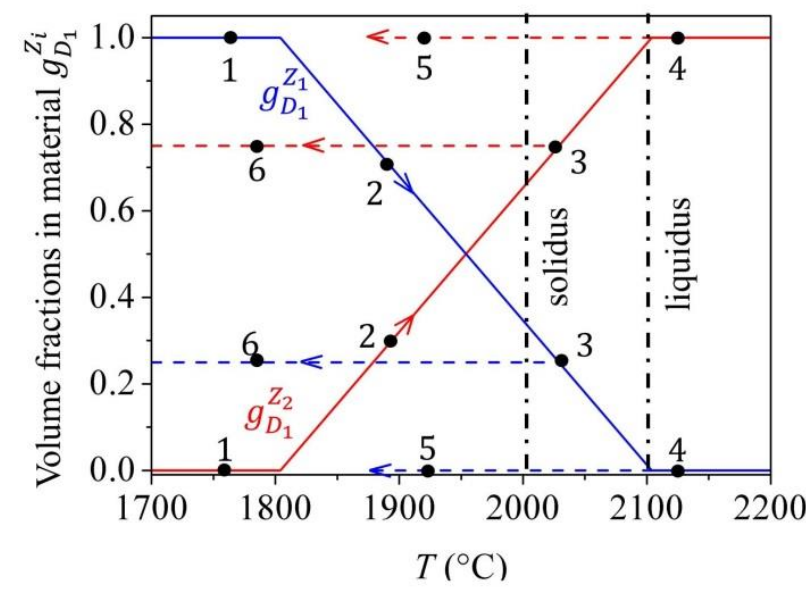

\begin{tabular}{|c|c|c|c|}
\hline State & $\mathbf{1}$ & $\mathbf{2 , 3 , 6}$ & $\mathbf{4 , 5}$ \\
\hline$g_{D_{1}}^{Z_{1}}$ & 1 & $>0$ & 0 \\
\hline$g_{D_{1}}^{Z_{2}}$ & 0 & $>0$ & 1 \\
\hline
\end{tabular}

$g_{D_{1}}^{Z_{1}}+g_{D_{1}}^{Z_{2}}=1$ (b)

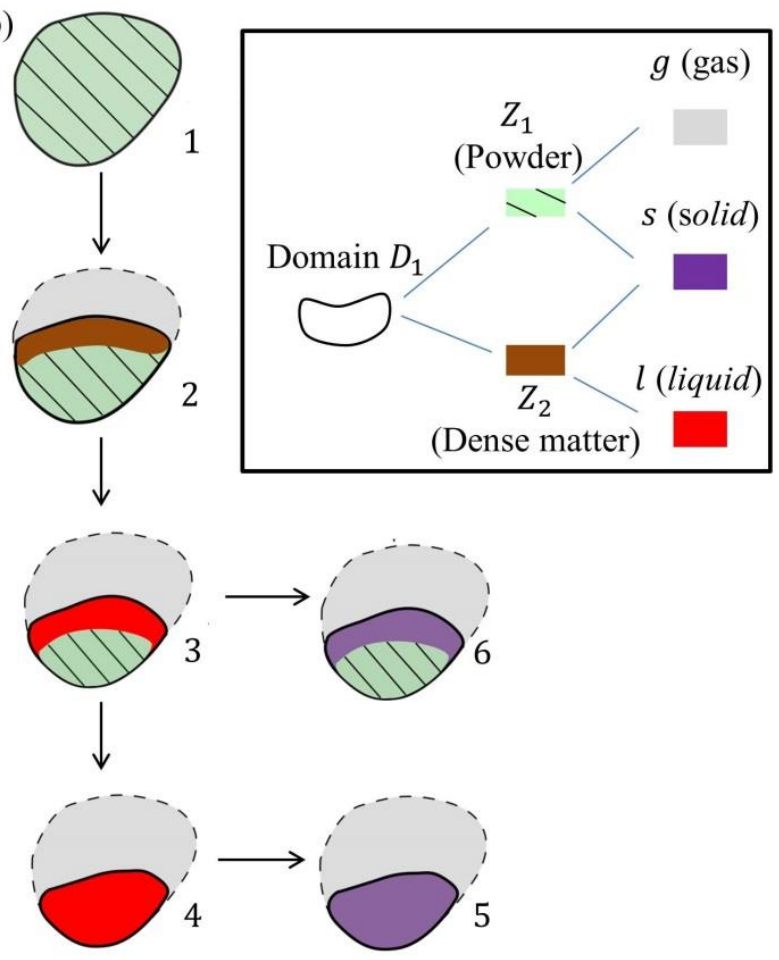

Figure 4: One-way transformation from powder (zone $Z_{1}$ ) to dense matter (zone $Z_{2}$ ) with (a) volume fractions as a function of temperature in domain $D_{1}$ and (b) illustration of possible distribution of zones and phases depending on the maximum heating temperature reached during the thermal history, (state 5) above and (state 6) below the melting temperature of the dense matter. Other states represent intermediate status of domain $D_{1}$ during the transformation path. 
(a)

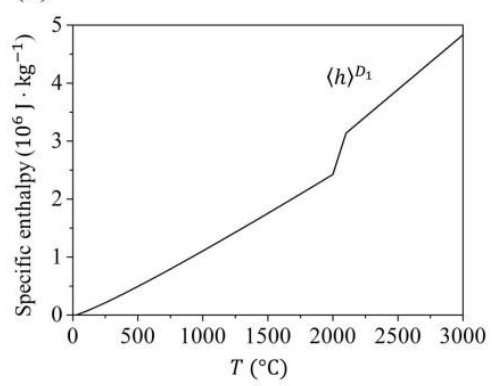

(b)

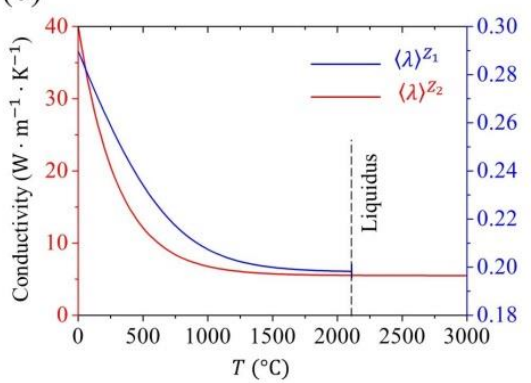

(c)

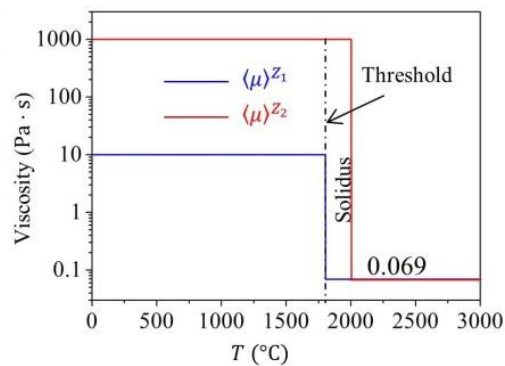

Figure 5: Temperature evolution of (a) the specific enthalpy in the whole domain $D_{1}$, (b) the thermal conductivity and (c) the dynamic viscosity of $\left(Z_{1}\right)$ powder and $\left(Z_{2}\right)$ dense matter. 


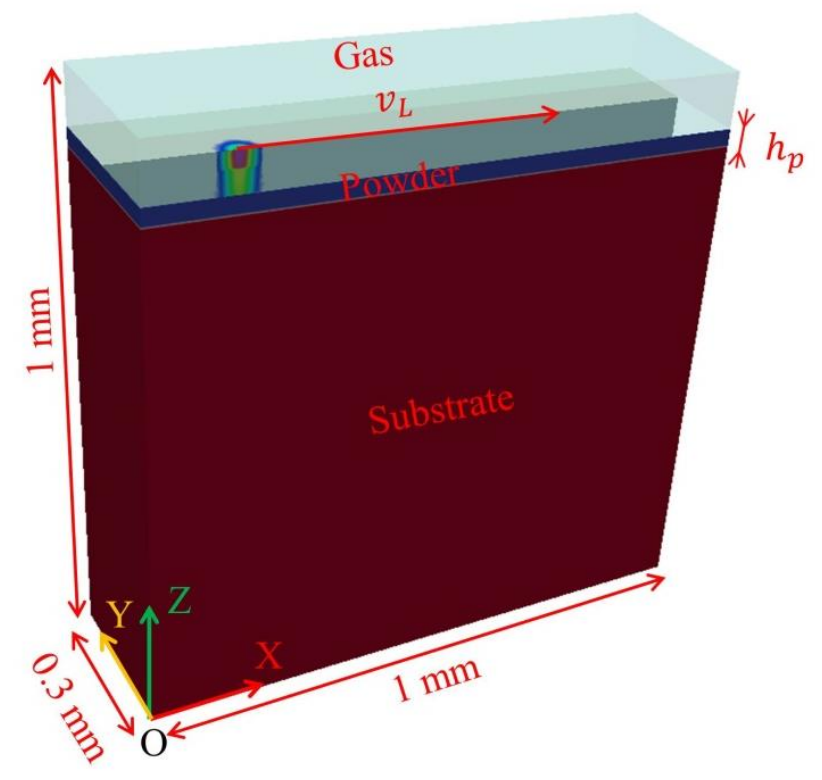

Figure 6: Configuration of unidirectional single pass simulation with overall dimensions, $(\mathrm{O}$, $\mathrm{X}, \mathrm{Y}, \mathrm{Z}$ ) frame and direction of the scanning velocity. Gas is with transparent rendering so as to reveal the initial position of the laser in a cut view at mid-width of the powder plus substrate sub-domains showing a multicolor temperature field. Different values for $h_{p}$ can be found in Table 2 (description of test cases). 

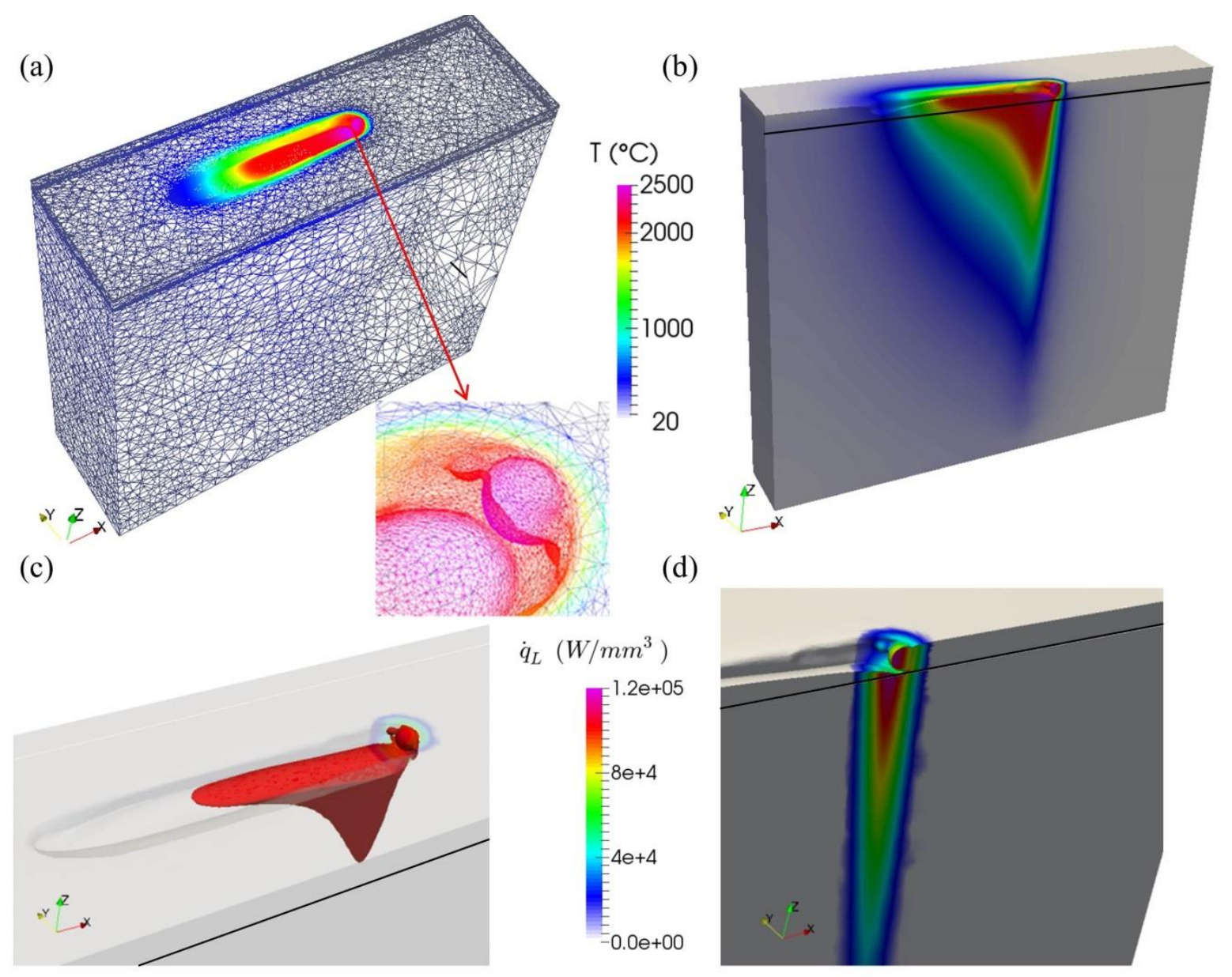

(d)

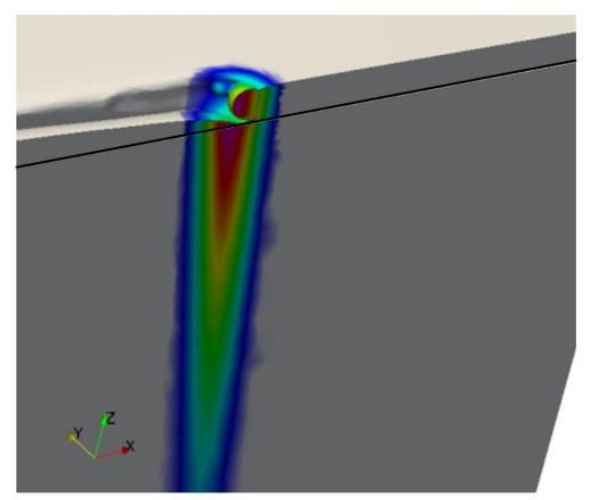

Figure 7: Snapshot for reference case at $t=2 \mathrm{~ms}$ illustrating (a) the FE mesh adaptation in the vicinity of the pool surface and $(a, b)$ temperature field (cut view along the vertical symmetry plane), (c) the melt pool defined by regions of temperature higher or equal to the melting temperature in the material domain (transparent rendering is active in order to show the whole melt pool), and (c, d) position of the heat source and distribution of the volumetric heat source term (cut view along the vertical symmetry plane). Gas is removed and the black line shows the powder/substrate boundary. 


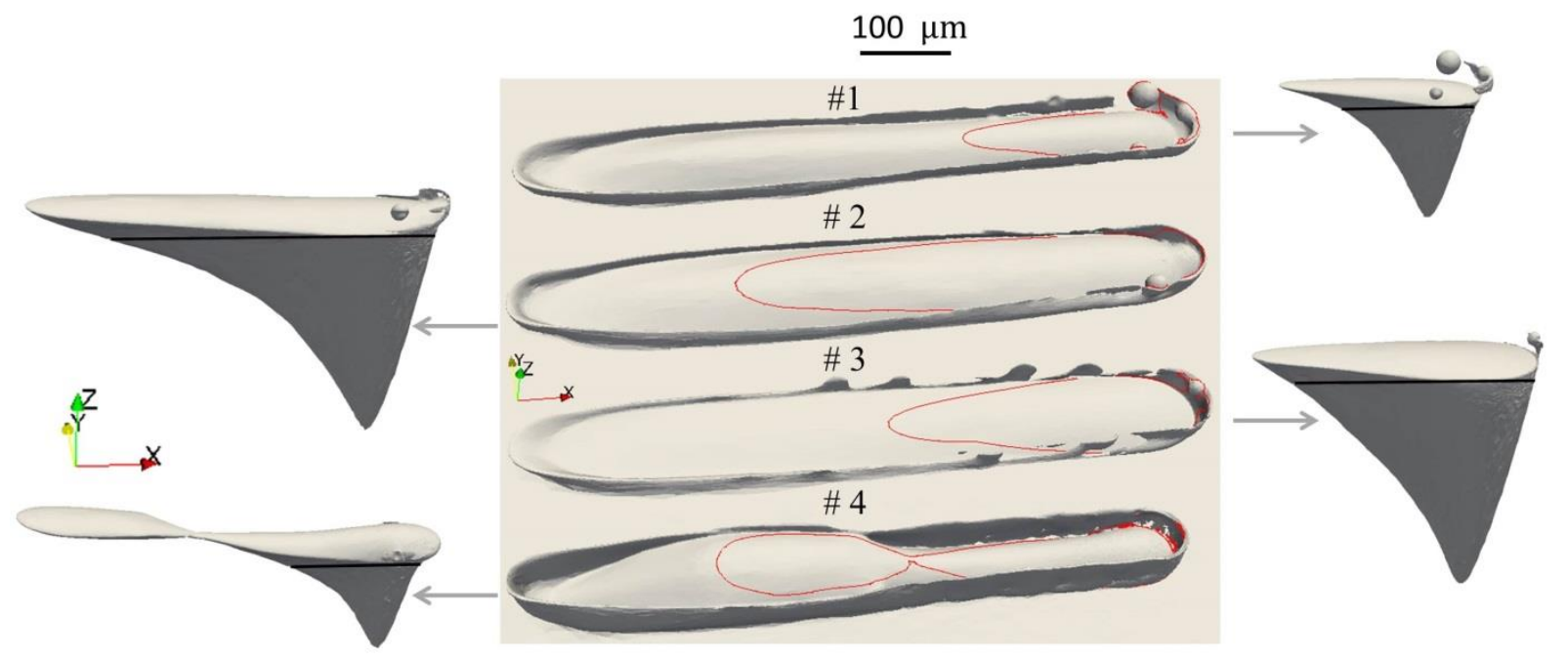

Figure 8: Simulated bead shape (center) and melt pool shape (two sides) for cases 1 4 at $t=3 \mathrm{~ms}$. Gas and powder are removed and the red contour indicates the melt pool. The skirt around the bead corresponds to powder partially melted and solidified. The black line is the powder/substrate boundary. 

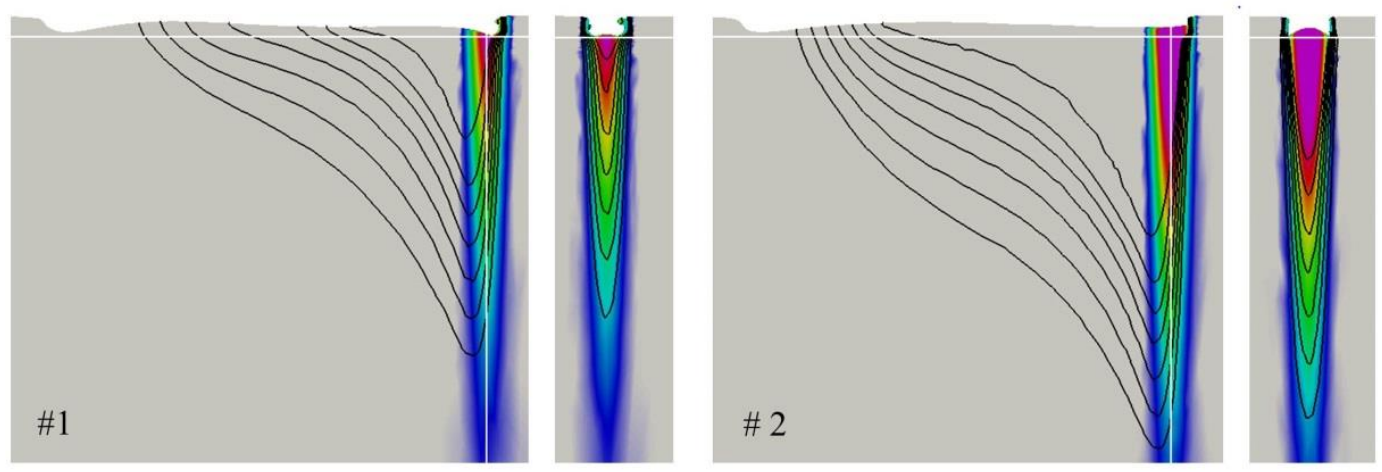

$\dot{q}_{L}\left(\mathrm{~W} \cdot \mathrm{mm}^{-3}\right)$
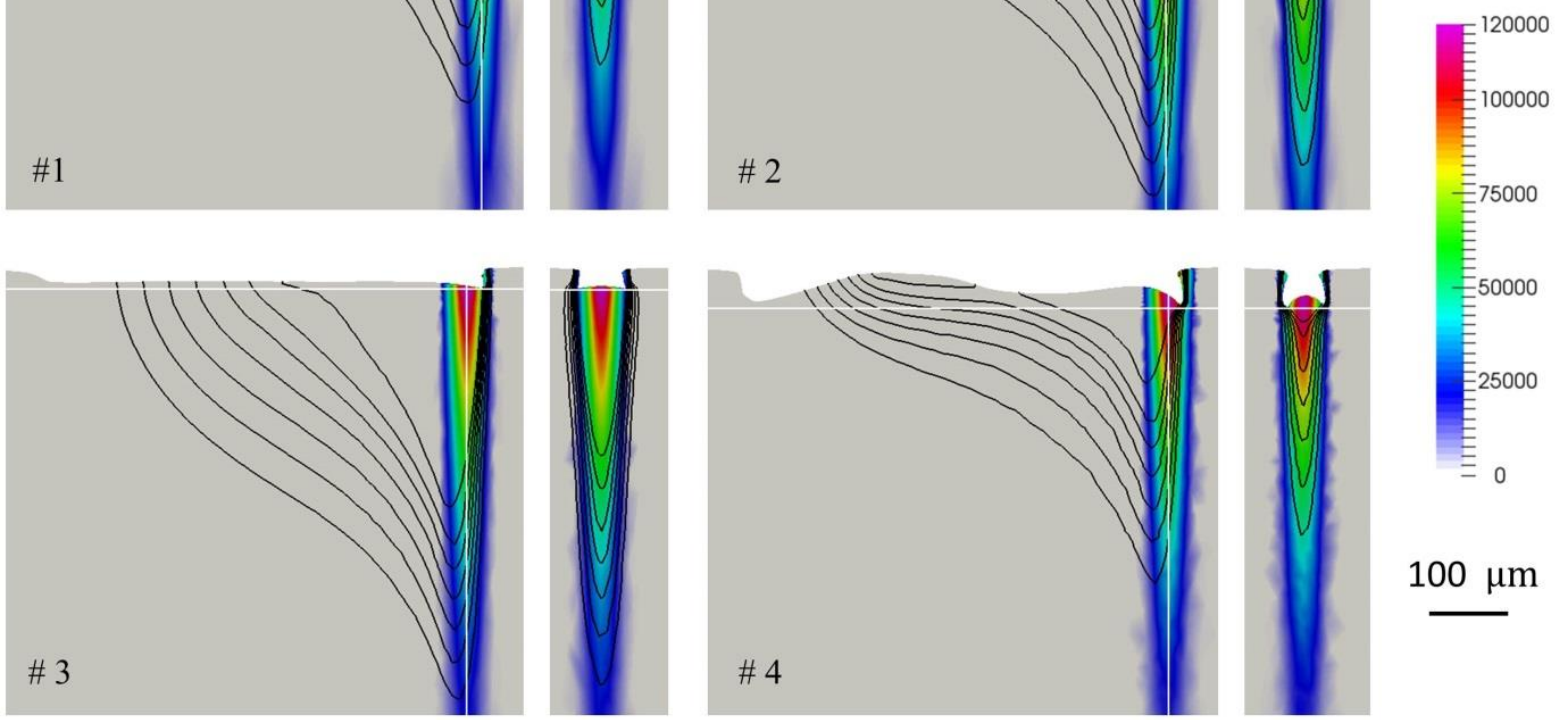

$100 \mu \mathrm{m}$

Figure 9: Longitudinal $(Y=0.15 \mathrm{~mm})$ and transversal $(X=0.6 \mathrm{~mm})$ section views of cases $1-4$ at $t=3 \mathrm{~ms}$. Black curves are temperature iso-contours in the range $\left[900{ }^{\circ} \mathrm{C}, 2100{ }^{\circ} \mathrm{C}\right]$ with $200{ }^{\circ} \mathrm{C}$ temperature steps, thus below the melting temperature, permitting a clear comparison of the melt pool depths. The white horizontal line is the powder/substrate boundary. The white vertical line in the longitudinal section indicates the position of the transversal section. 
(a)

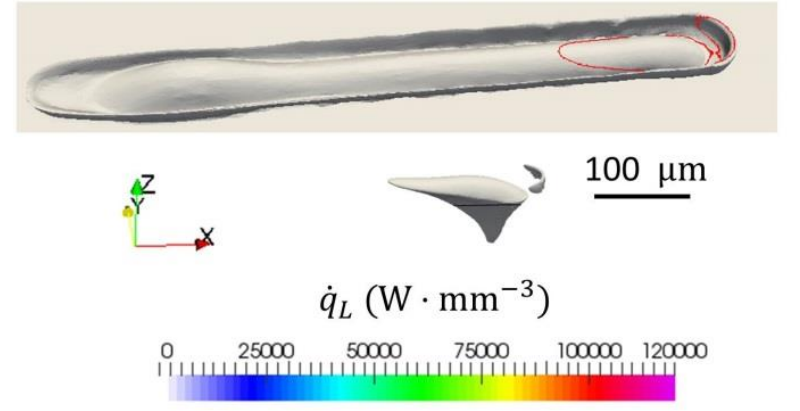

(b) $100 \mu \mathrm{m}$

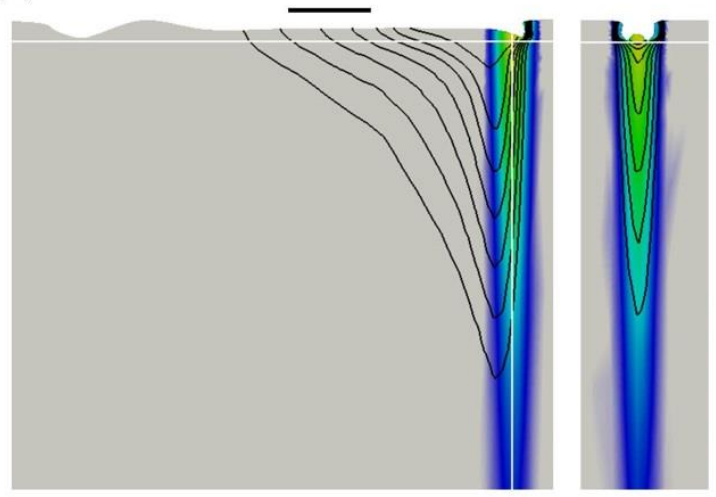

Figure 10: Displays from the analysis of the case 5 simulation showing (a) the shape of the bead and the melt pool at $t=3 \mathrm{~ms}$ with the same scale as in Figure 8, and (b) the heat source distribution and temperature iso-contours with the same scale as in Figure 9. 
(a)

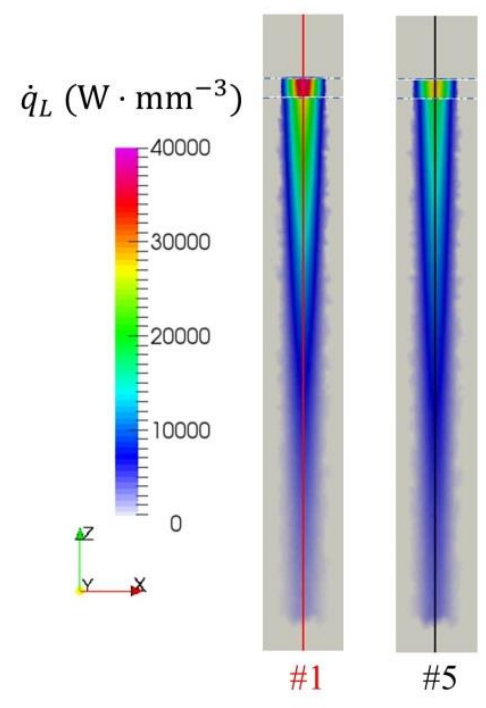

(b)

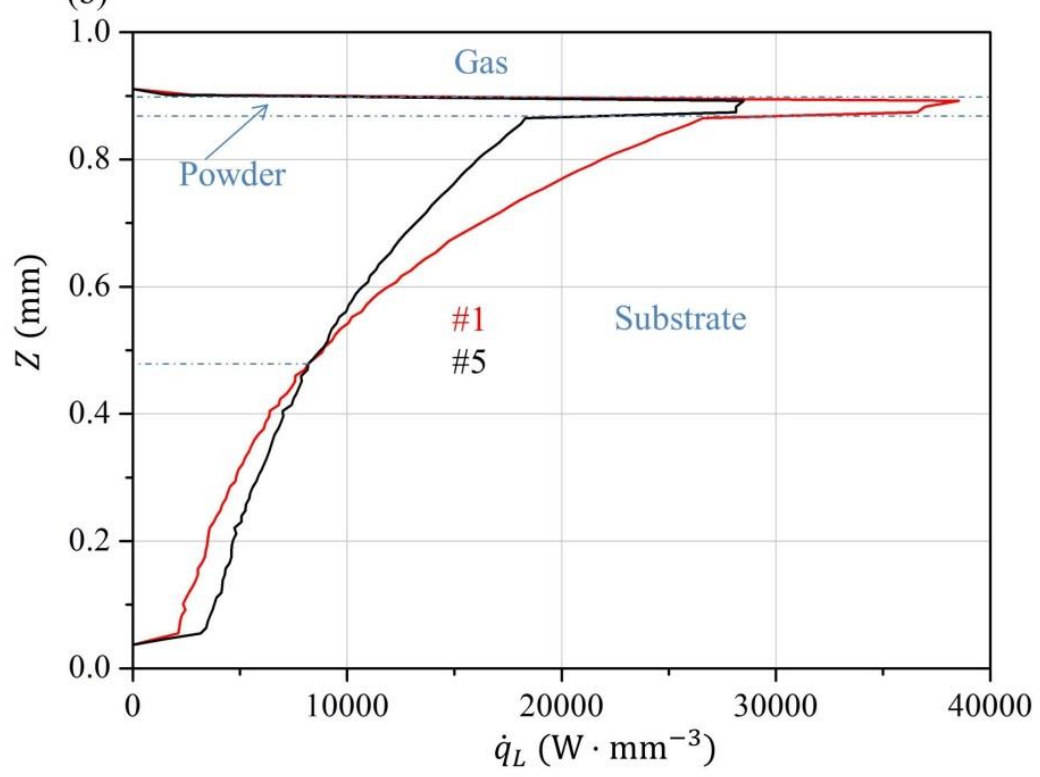

Figure 11: (a) Heat source distribution and (b) profile along laser axe of case 1 (red) and 5 (black) with different absorption coefficient at $t=4 \mu$ s (corresponding to $24.6 \%$ of $P_{L}(1-$ $R$ ) imposed). 


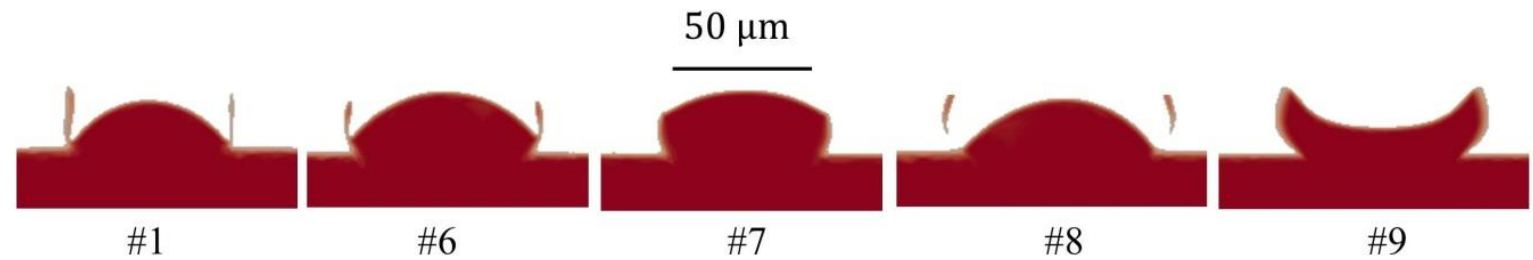

Figure 12: Transversal cross sections at $X=0.5 \mathrm{~mm}, t=2 \mathrm{~ms}$ of case $1,6,7,8$ and 9. Gas and powder are removed. 


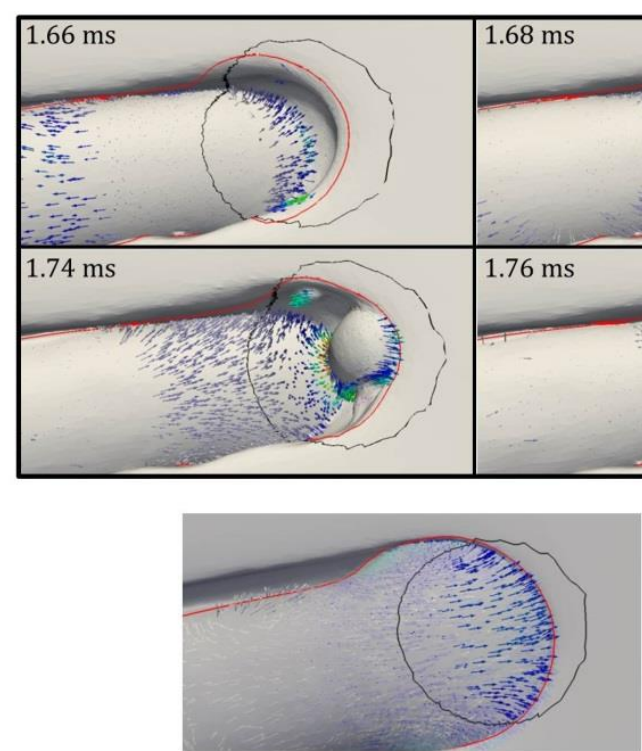

\#8

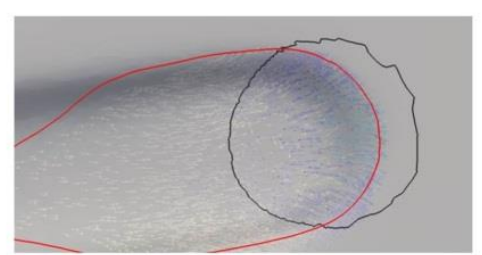

$\# 9$

Figure 13: Comparison of melt pool of cases 1, 8 and 9 with different value of the surface tension. The melt pool (red, iso-contour of liquidus) and the laser spot (black contour) with $R_{L}=35 \mu \mathrm{m}$ are highlighted. The surface in cases 8 and 9 is drawn with transparency in order to better show the velocity field as the fluid flows is principally developed below the surface. 

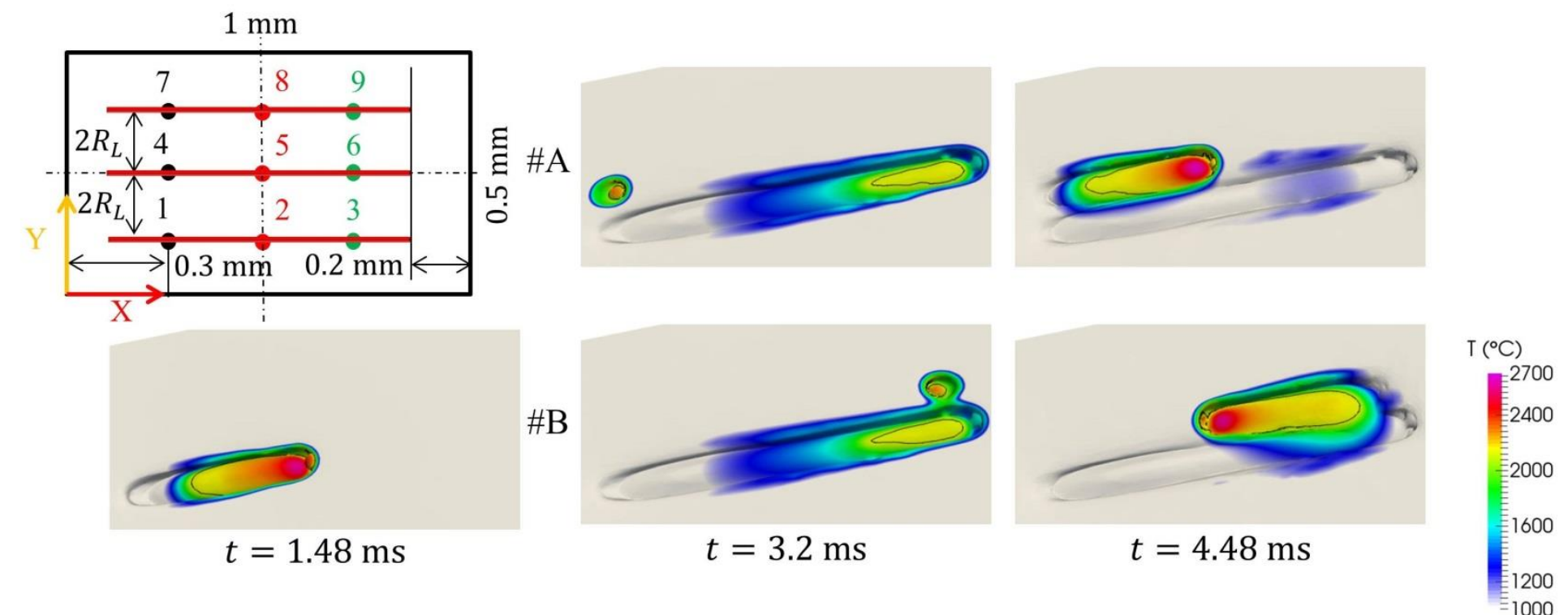

\#A
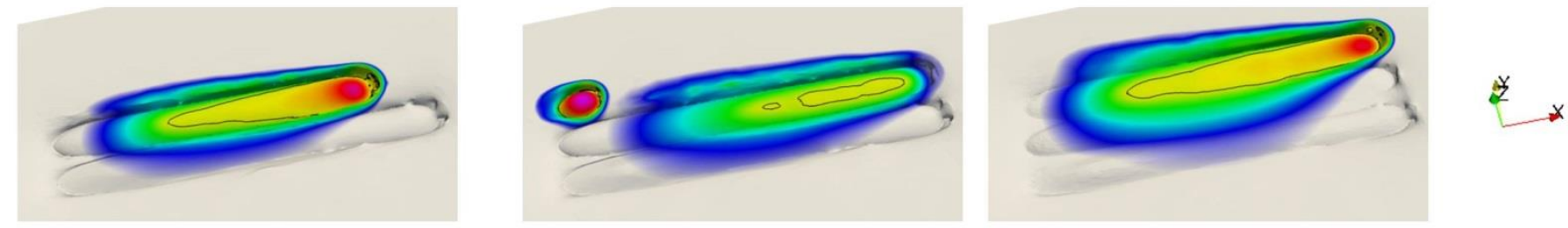

\#B

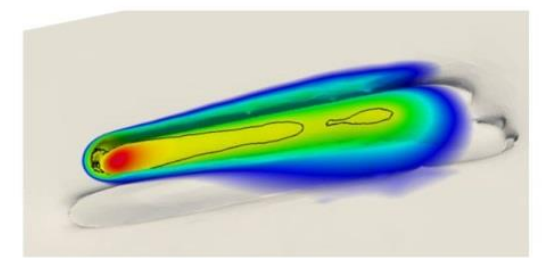

$$
t=5.6 \mathrm{~ms}
$$

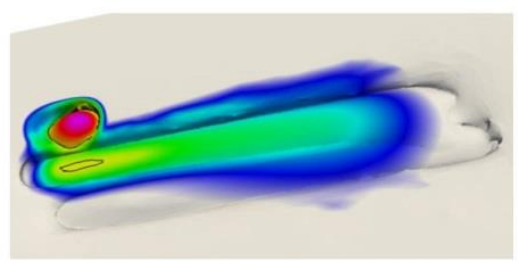

$t=6.4 \mathrm{~ms}$

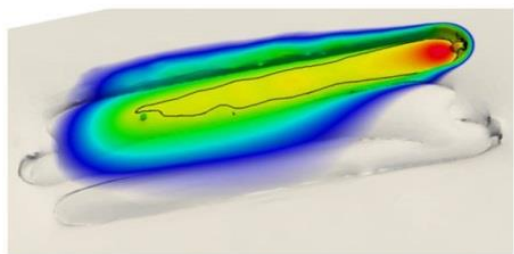

$t=9 \mathrm{~ms}$

Figure 14: Temperature field at different times during 3 passes with (\#A) unidirectional scanning and (\#B) alternate scanning. The black contour is the melt pool. Temperature range is scaled in $400 \sim 2700^{\circ} \mathrm{C}$ in order to have a good contrast. 

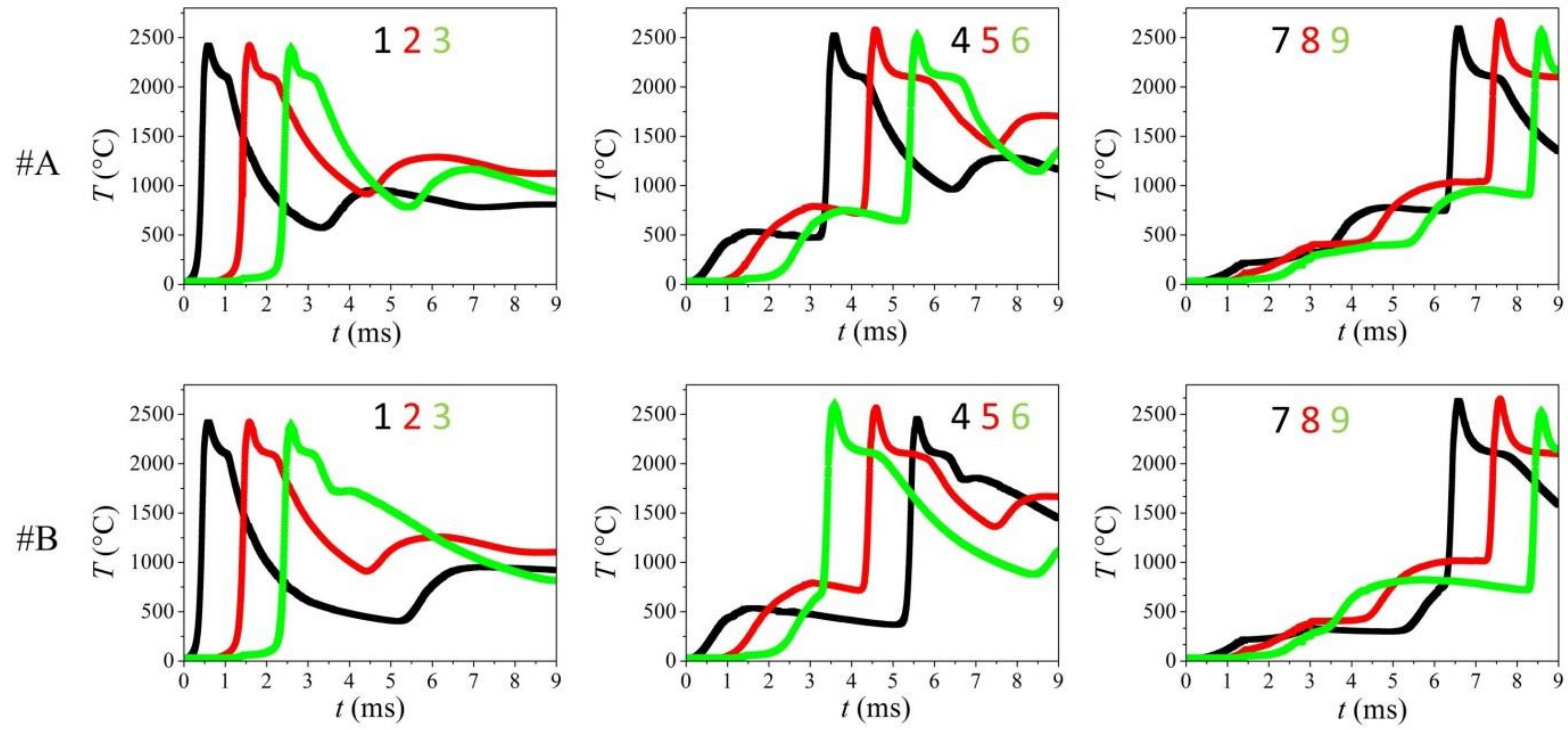

Figure 15: Temperature evolution of local points 1 9 for (\#A) unidirectional scanning and (\#B) alternate scanning. 